Document downloaded from:

http://hdl.handle.net/10251/99749

This paper must be cited as:

Bès, JP.; Menet, Q.; Peris Manguillot, A.; Puig-De Dios, Y. (2016). Recurrence properties of hypercyclic operators. Mathematische Annalen. 366(1):545-572. doi:10.1007/s00208-0151336-3

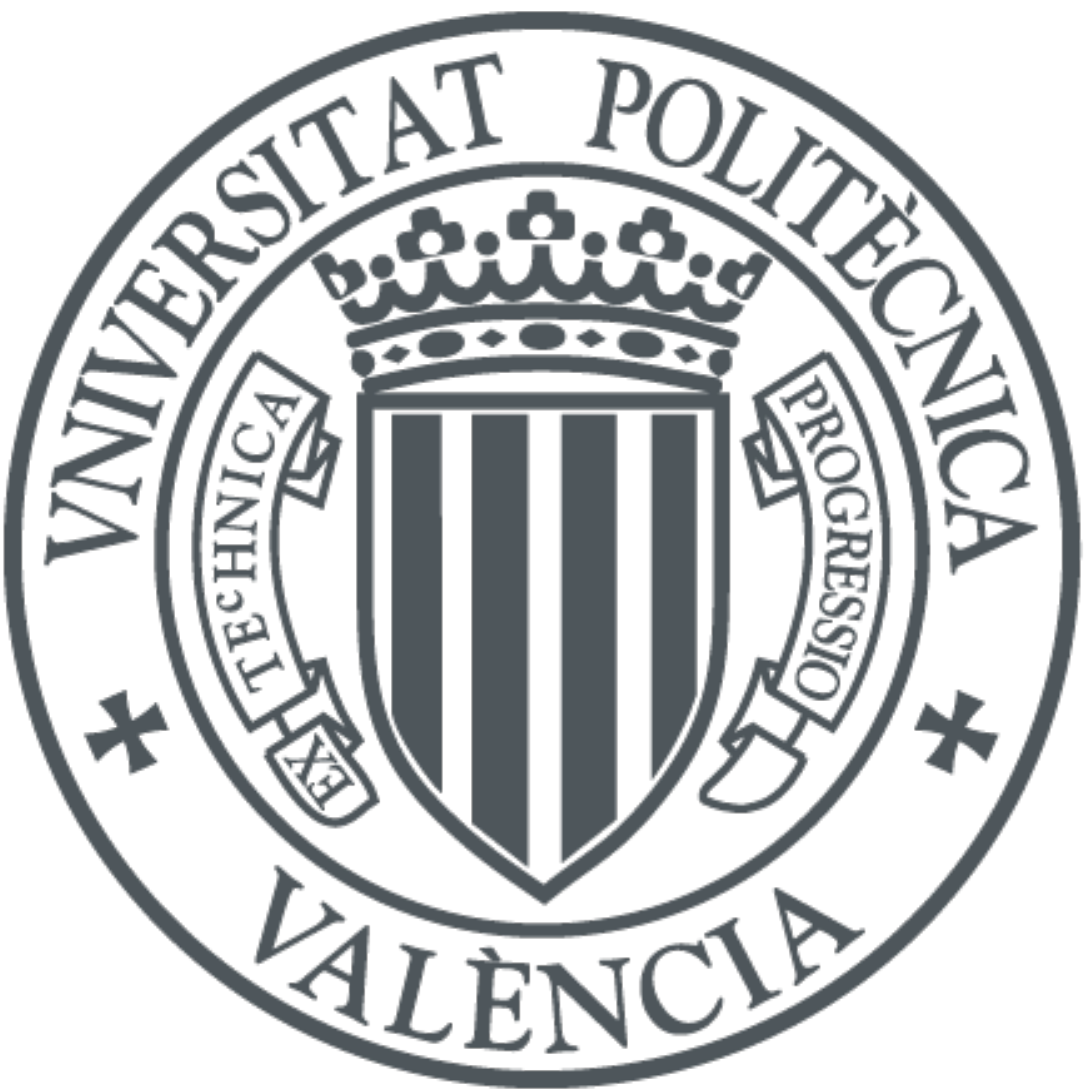

The final publication is available at

http://doi.org/10.1007/s00208-015-1336-3

Copyright Springer-Verlag

Additional Information 


\section{Recurrence properties of hypercyclic operators}

Article in Mathematische Annalen · October 2014

DOI: 10.1007/s00208-015-1336-3 · Source: arXiv

CITATIONS

10

\section{4 authors:}

\section{J. Bès}

Bowling Green State University

25 PUBLICATIONS 556 CITATIONS

SEE PROFILE

\section{Alfred Peris}

Universitat Politècnica València 110 PUBLICATIONS 1,956 CITATIONS

SEE PROFILE
READS

175
Quentin Menet

Université d'Artois

22 PUBLICATIONS 98 CITATIONS

SEE PROFILE

Yunied Puig de Dios

University of California, Riverside

5 PUBLICATIONS 12 CITATIONS

SEE PROFILE

Some of the authors of this publication are also working on these related projects: 


\title{
Recurrence properties of hypercyclic operators *
}

\author{
J. Bès † Q. Menet $;$ A. Peris ${ }^{\S}$ and Y. Puigq
}

\begin{abstract}
We generalize the notions of hypercyclic operators, $\mathfrak{U}$-frequently hypercyclic operators and frequently hypercyclic operators by introducing a new notion of hypercyclicity, called $\mathcal{A}$-frequent hypercyclicity. We then state an $\mathcal{A}$-Frequent Hypercyclicity Criterion, inspired from the Hypercyclicity Criterion and the Frequent Hypercyclicity Criterion, and we show that this criterion characterizes the $\mathcal{A}$-frequent hypercyclicity for weighted shifts. We finish by investigating which kind of properties of density can have the sets $N(x, U)=\left\{n \in \mathbb{N}: T^{n} x \in U\right\}$ for a given hypercyclic operator and study the new notion of reiteratively hypercyclic operators.
\end{abstract}

\section{Introduction}

Our purpose is to study how 'often' the orbit of a vector under a linear operator can meet arbitrary non-empty open sets. The (chaotic) dynamics of linear operators is a very active topic of research, and the books [4] and [16] contain many of the recent advances.

Let $X$ be a separable $F$-space (i.e., a metrizable and complete topological vector space), $L(X)$ the space of continuous linear operators on $X$, and $T \in L(X)$. Given $x \in X$, its orbit under $T$ is $\operatorname{Orb}(x, T)=\left\{x, T x, T^{2} x, \ldots\right\}$. We denote by $\mathbb{N}$ the set of positive integers, by $\mathbb{Z}_{+}$the set of non-negative integers and for any $x \in X$, any subset $V \subset Y$, we denote the times set of $\operatorname{Orb}(x, T)$ hitting $V$ by

$$
N(x, V)=\left\{n \geq 0: T^{n} x \in V\right\} .
$$

Given $A \subseteq \mathbb{Z}_{+}$, its upper and lower densities are defined, respectively, by

$$
\bar{d}(A)=\limsup _{k \rightarrow \infty} \frac{|A \cap[0, k]|}{k} \text { and } \quad \underline{d}(A)=\liminf _{k \rightarrow \infty} \frac{|A \cap[0, k]|}{k} .
$$

The operator $T$ is said to be hypercyclic if there exists $x \in X$ such that for any nonempty open set $V \subset X, N(x, V)$ is non-empty (or, equivalently since $X$ has no isolated

\footnotetext{
*This work is supported in part by MICINN and FEDER, Project MTM2013-47093-P, and by GVA, Project PROMETEOII/2013/013. The second author is supported by a grant of FRIA.

$\dagger$ Department of Mathematics and Statistics, Bowling Green State University, Bowling Green, OH 43403, USA. e-mail:jbes@math.bgsu.edu

${ }^{\ddagger}$ Département de Mathématique, Université de Mons, 20 Place du Parc, 7000 Mons, Belgique. e-mail: Quentin.Menet@umons.ac.be

§IUMPA, Universitat Politècnica de València, Departament de Matemàtica Aplicada, Edifici 7A, 46022 València, Spain. e-mail: aperis@mat.upv.es

"Università degli studi di Milano, Dipartimento di Matematica "Federigo Enriques", Via Saldini 50 20133 Milano, Italy. e-mail: yunied.puig@unimi.it
} 
points, infinite). It is $\mathfrak{U}$-frequently hypercyclic if there exists $x \in X$ such that for any nonempty open set $V, N(x, V)$ is a set of positive upper density, and it is frequently hypercyclic if there exists $x \in X$ such that for any non-empty open set $V \subset Y, N(x, V)$ is a set of positive lower density. Frequently hypercyclic operators were introduced by Bayart and Grivaux [2] while $\mathfrak{U}$-frequently hypercyclic operators were introduced by Shkarin [20].

We generalize these notions of hypercyclicity as follows:

Definition 1. Let $\mathcal{A} \subset \mathcal{P}\left(\mathbb{Z}_{+}\right)$be a non-trivial hereditarily upward family of subsets of $\mathbb{Z}_{+}$(i.e., $\emptyset \notin \mathcal{A}$ and for any $A \in \mathcal{A}$, if $A \subset B$, then $B \in \mathcal{A}$ ). If, moreover,

$\left(^{*}\right) \mathcal{A}$ contains a sequence $\left(A_{k}\right)$ of disjoint sets such that for any $j \in A_{k}$, any $j^{\prime} \in A_{k^{\prime}}$, $j \neq j^{\prime}$, we have $\left|j^{\prime}-j\right| \geq \max \left\{k, k^{\prime}\right\}$,

then we say that $\mathcal{A}$ is a hypercyclicity set. Given a non-trivial hereditarily upward family $\mathcal{A} \subset \mathcal{P}\left(\mathbb{Z}_{+}\right)$and $T \in L(X)$, the operator $T$ is called $\mathcal{A}$-frequently hypercyclic if there exists $x \in X$ such that for any non-empty open set $V \subset X, N(x, V) \in \mathcal{A}$. Such a vector $x$ is called an $\mathcal{A}$-frequently hypercyclic vector for $T$.

An operator $T$ is thus hypercyclic, $\mathfrak{U}$-frequently hypercyclic or frequently hypercyclic if it is $\mathcal{A}$-frequently hypercyclic, respectively, for $\mathcal{A}_{\infty}$ (the family of infinite subsets of $\mathbb{Z}_{+}$), for the set of positive upper density sets, or for the set of positive lower density sets. This concept also generalizes the notions of $\left(m_{k}\right)$-hypercyclic operators introduced by Bayart and Matheron [5].

For hypercyclic operators and frequently hypercyclic operators, we have at our disposal the well-known Hypercyclicity Criterion [9] and the Frequent Hypercyclicity Criterion [10]. We generalize these criteria to the framework of $\mathcal{A}$-frequently hypercyclic operators (Section 2.1). In particular, we obtain a $\mathfrak{U}$-Frequent Hypercyclicity Criterion. The $\mathcal{A}$-Frequent Hypercyclicity Criterion also improves the Frequent Hypercyclicity Criterion. Indeed, we know that the Frequent Hypercyclicity Criterion does not characterize frequently hypercyclic operators because Bayart and Grivaux [3] have exhibited a frequently hypercyclic weighted shift on $c_{0}$ that is neither chaotic nor mixing, whereas if $T$ satisfies the Frequent Hypercyclicity Criterion, then $T$ is mixing and chaotic. However, we succeed to show that, in the case of weighted shifts on $\ell^{p}$ or $c_{0}$, the $\mathcal{A}$-Frequent Hypercyclicity Criterion characterizes the $\mathcal{A}$-frequent hypercyclicity (Section 2.2) and thus, in particular, an operator $T$ can satisfy the $\mathcal{A}$-Frequent Hypercyclicity Criterion even if it is neither chaotic nor mixing. This characterization of $\mathcal{A}$-frequently hypercyclic weighted shifts complements the characterization of hypercyclic weighted shifts [13, 18] and the recent characterization of frequently hypercyclic weighted shifts on $\ell^{p}$ and $c_{0}[6]$.

In Section 3 we will concentrate in $\mathcal{A}$-frequently hypercyclic operators for families $\mathcal{A}$ with positive Banach densities.

Definition 2. Let $A \subseteq \mathbb{Z}_{+}, \alpha^{s}:=\limsup _{k \rightarrow \infty}|A \cap[k+1, k+s]|$ and $\alpha_{s}:=\liminf _{k \rightarrow \infty} \mid A \cap$ $[k+1, k+s] \mid$. The upper and lower Banach densities are defined respectively by

$$
\overline{B d}(A)=\lim _{s \rightarrow \infty} \frac{\alpha^{s}}{s} \quad \text { and } \quad \underline{B d}(A)=\lim _{s \rightarrow \infty} \frac{\alpha_{s}}{s} .
$$

The above densities are well-defined for any set $A \subseteq \mathbb{Z}_{+}$. A proof of this fact can be found in [19] and we have

$$
\underline{B d}(A) \leq \underline{d}(A) \leq \bar{d}(A) \leq \overline{B d}(A) .
$$


For any choice of real numbers $0 \leq r_{1} \leq r_{2} \leq r_{3} \leq r_{4} \leq 1$, one can even find sets $A$ such that

$$
\underline{B d}(A)=r_{1}, \underline{d}(A)=r_{2}, \bar{d}(A)=r_{3}, \overline{B d}(A)=r_{4} .
$$

This result has been recently announced, and will appear in a joint paper by G. Grekos, R. Jin and L. Mišík.

Suppose we want to classify and study all the recurrence properties appearing when $N(x, U)$ has a lower or upper (Banach) density greater than 0 or equal to 1 . We show that only the following properties make sense.

- $\underline{d}(N(x, U))>0$, i.e. $T$ is frequently hypercyclic

- $\bar{d}(N(x, U))>0$, i.e. $T$ is $\mathfrak{U}$-frequently hypercyclic

- $\overline{B d}(N(x, U))>0$, i.e. $T$ is reiteratively hypercyclic.

We then focus on the new notion of reiteratively hypercyclic operators. Obviously, frequently hypercyclic operators are $\mathfrak{U}$-frequently hypercyclic and these in turn are reiteratively hypercyclic. In a recent article, Bayart and Ruzsa have shown the existence of a $\mathfrak{U}$-frequently hypercyclic weighted backward shift on $c_{0}$ which is not frequently hypercyclic, see [6, Theorem 5]. We complement this result by showing that reiterative hypercyclicity is not equivalent to $\mathfrak{U}$-frequent hypercyclicity. More precisely, thanks to the characterization of $\mathcal{A}$-frequently hypercyclic weighted shifts, we show that there exists a reiteratively hypercyclic weighted backward shift on $c_{0}$ which is not $\mathfrak{U}$-frequently hypercyclic (Theorem 17). On the other hand, Bayart and Ruzsa proved that frequent hypercyclicity and $\mathfrak{U}$-frequent hypercyclicity are equivalent notions for backward shifts on $\ell^{p}(\mathbb{Z})$ and $\ell^{p}\left(\mathbb{Z}_{+}\right)$, see [6, Theorems 3-4]. We generalize this equivalence by showing that every reiteratively hypercyclic weighted shift on $\ell^{p}(\mathbb{Z})$ or on $\ell^{p}\left(\mathbb{Z}_{+}\right)$is frequently hypercyclic (Theorem 23). In particular, we deduce that there exists some mixing operator which is not reiteratively hypercyclic. Finally, we observe that any reiteratively hypercyclic operator is topologically ergodic, i.e.

$$
N(U, V):=\left\{n \geq 0 ; T^{n} U \cap V \neq \emptyset\right\}
$$

is syndetic (has bounded gaps) for any $U, V$ non-empty open sets. Since topologically ergodic operators are weakly mixing [15], we deduce that any reiteratively hypercyclic operator $T$ is weakly mixing.

\section{$2 \mathcal{A}$-frequently hypercyclic operators}

The definition of $\mathcal{A}$-frequently hypercyclic operators justifies the property $\left.{ }^{*}\right)$ of hypercyclicity sets (Definition 1). Indeed, the fact that $\mathcal{A}$ is non-trivial provides that, for any non-empty open set $V, N(x, V)$ is at least non-empty. The hereditarily upward condition implies that, for any non-empty open sets $U \subset V$, if $N(x, U) \in \mathcal{A}$, then $N(x, V) \in \mathcal{A}$. In particular, if $X$ is a topological vector space with a countable open basis $\left(U_{n}\right)_{n \geq 1}$, we deduce that in order to prove that a vector $x$ is $\mathcal{A}$-frequently hypercyclic, it is sufficient to prove that $N\left(x, U_{n}\right) \in \mathcal{A}$ for each $n \geq 1$. Finally, condition $\left(^{*}\right)$ is necessary for the existence of $\mathcal{A}$-frequently hypercyclic operators on Banach spaces.

Proposition 3. Let $X$ be a separable Banach space, $X \neq\{0\}$, let $\mathcal{A} \subset \mathcal{P}\left(\mathbb{Z}_{+}\right)$be a nontrivial hereditarily upward family, and let $T \in \mathcal{L}(X)$. If $T$ is $\mathcal{A}$-frequently hypercyclic, then $\mathcal{A}$ is a hypercyclicity set. 
Proof. Since $X \neq\{0\}$ and $T$ is $\mathcal{A}$-frequently hypercyclic, the norm of $T$ is bigger than 1 . Let $K=\|T\|>1$ and $x$ an $\mathcal{A}$-frequently hypercyclic vector for $T$. We first show that for any $n \geq 1$, any $C>0$, there exist $y \in X$ and $\varepsilon<1$ such that if $A:=N(x, B(0, C))$ and $B:=N(x, B(y, \varepsilon))$, then $d(A, B) \geq n$ and for any $j \neq j^{\prime} \in B,\left|j-j^{\prime}\right| \geq n$.

If $j \in A$, then $\left\|T^{j} x\right\|<C$ and we have $\left\|T^{i} x\right\|<C K^{n-1}$ for any $j \leq i \leq j+n-1$. In particular, if $\|y\|>C K^{n-1}+1$, we deduce that for any $j \leq i \leq j+n-1$, we have $i \notin B$. Therefore, we consider a vector $y \in \operatorname{Orb}(x, T)$ such that $\|y\|>C K^{n-1}+1$ and such that for any $0 \leq i \leq n-1,\left\|T^{i} y\right\|>C+K^{n-1}$. Such a vector exists because otherwise the orbit of $x$ would not be dense. We thus have that for any $0 \leq i \leq n-1$, if $j \in B$, then

$$
\left\|T^{j+i} x\right\| \geq\left\|T^{i} y\right\|-\left\|T^{i}\left(T^{j} x-y\right)\right\|>\left(C+K^{n-1}\right)-K^{n-1}\left\|T^{j} x-y\right\| \geq C .
$$

We deduce that for any $0 \leq i \leq n-1$, if $j \in B$, then $j+i \notin A$ and we thus conclude that $d(A, B) \geq n$.

Now, since $y \in \operatorname{Orb}(x, T)$, the vector $y$ is not periodic and there thus exists $0<\eta \leq 1$ such that for any $1 \leq i \leq n-1,\left\|T^{i} y-y\right\|>\eta$. If we let $\varepsilon:=\frac{\eta}{1+K^{n-1}}$, then we have for any $j \in B$, any $1 \leq i \leq n-1$,

$$
\left\|T^{j+i} x-y\right\| \geq\left\|T^{i} y-y\right\|-\left\|T^{i}\left(T^{j} x-y\right)\right\|>\eta-K^{n-1} \varepsilon \geq \varepsilon .
$$

Hence, for any $j, j^{\prime} \in B, j \neq j^{\prime}$, we have $\left|j-j^{\prime}\right| \geq n$.

We conclude that there exists a sequence $\left(y_{k}\right) \subset X$, an increasing sequence $\left(C_{k}\right)$ and a sequence $\left(\varepsilon_{k}\right)$ such that if $A_{k}:=N\left(x, B\left(0, C_{k}\right)\right)$ and $B_{k}:=N\left(x, B\left(y_{k}, \varepsilon_{k}\right)\right)$, then for any $k \geq 1, d\left(A_{k}, B_{k}\right) \geq k, B_{k} \subset A_{k+1}$ and for any $j \neq j^{\prime} \in B_{k},\left|j-j^{\prime}\right| \geq k$. Since the sequence $\left(A_{k}\right)$ is increasing, we deduce that the sequence $\left(B_{k}\right) \subset \mathcal{A}$ satisfies the desired property. Indeed, if $j \in B_{k}, j^{\prime} \in B_{k^{\prime}}$ and $k>k^{\prime}$, then we know that $j^{\prime} \in A_{k}$ and thus $\left|j-j^{\prime}\right| \geq k$, and if $j, j^{\prime} \in B_{k}$ with $j \neq j^{\prime}$, we know that $\left|j-j^{\prime}\right| \geq k$ by definition of $B_{k}$.

Nevertheless, if $X$ is not a Banach space, it is possible that there exist a family $\mathcal{A}$ and an operator $T$ on $X$ such that $T$ is $\mathcal{A}$-frequently hypercyclic and $\mathcal{A}$ is not a hypercyclicity set.

Example 4. Let $\phi: \mathbb{N} \rightarrow \mathbb{N}$ such that for any $i, j \geq 1$, there exists $n \geq 1$ satisfying $(\phi(n+1), \phi(n+2))=(i, j)$. For any $k \geq 1$, we let $\psi(k)=\sum_{l=1}^{k} \phi(l), A_{k}=\{\psi(n)$ : $\phi(n+1)=k\}$ and $\mathcal{A}=\left\{B: B \supseteq A_{k}\right.$ for some $\left.k\right\}$. Since $d\left(A_{k}, A_{k^{\prime}}\right)=\min \left(k, k^{\prime}\right)$, we deduce that $\mathcal{A}$ does not contain a sequence $\left(B_{k}\right)$ of disjoint sets such that for any $j \in B_{k}$, any $j^{\prime} \in B_{k^{\prime}}, j \neq j^{\prime}$, we have $\left|j^{\prime}-j\right| \geq \max \left\{k, k^{\prime}\right\}$. Nevertheless, if we consider the forward shift $S$ on $\omega$ and a dense sequence $\left(x_{k}\right)_{k \geq 1} \subset \omega$ such that for any $k \geq 1$,

$$
k>d\left(x_{k}\right):=\sup \left\{i \geq 0: x_{k}(i) \neq 0\right\},
$$

then the vector $x=\sum_{n=1}^{\infty} S^{\psi(n)} x_{\phi(n+1)}$ is an $\mathcal{A}$-frequently hypercyclic vector for the backward shift on $\omega$.

\section{$2.1 \mathcal{A}$-Frequent Hypercyclicity Criterion}

For the hypercyclic operators and the frequently hypercyclic operators on $F$-spaces, we have at our disposal the well-known Hypercyclicity Criterion [9] and the Frequent Hypercyclicity Criterion [10]. We show in this section how to generalize these criteria to the notion of $\mathcal{A}$-frequent hypercyclicity.

We recall that a $F$-space is a topological vector space whose the topology is induced by a complete translation-invariant metric. In fact, if $X$ is a $F$-space, there exists a complete translation-invariant metric $d$ such that $\|x\|=d(x, 0)$ is a $F$-norm. 
Definition 5. Let $X$ be a vector space. A map $\|\cdot\|$ from $X$ to $\mathbb{R}^{+}$is a $F$-norm if for any $x, y \in X$, any $\lambda \in \mathbb{K}$,

1. $\|x+y\| \leq\|x\|+\|y\|$

2. $\|\lambda x\| \leq\|x\|$ if $|\lambda|<1$

3. $\lim _{\lambda \rightarrow 0}\|\lambda x\|=0$;

4. $\|x\|=0$ implies $x=0$.

An interesting property about $F$-norms following from 1 . and 2 . is that for any $x \in X$, any $\lambda \in \mathbb{K}$, we have

$$
\|\lambda x\| \leq(|\lambda|+1)\|x\| .
$$

The Hypercyclicity Criterion and the Frequent Hypercyclicity Criterion can be stated as follows.

Theorem 6 (Hypercyclicity Criterion [9]). Let $X$ be a separable $F$-space and $T \in \mathcal{L}(X)$. If there are dense subsets $X_{0} \subset X, Y_{0} \subset X$, an increasing sequence $\left(n_{k}\right)_{k \geq 1}$ of positive integers and maps $S_{n_{k}}: Y_{0} \rightarrow X$, such that for any $x \in X_{0}, y \in Y_{0}$,

1. $T^{n_{k}} x \rightarrow 0$,

2. $S_{n_{k}} y \rightarrow 0$,

3. $T^{n_{k}} S_{n_{k}} y \rightarrow y$,

then $T$ is weakly mixing and thus hypercyclic.

Theorem 7 (Frequent Hypercyclicity Criterion [10]). Let $X$ be a separable F-space and $T \in \mathcal{L}(X)$. If there are a dense subset $Y_{0} \subset Y$ and $S_{n}: Y_{0} \rightarrow X, n \geq 0$ such that for each $y \in Y_{0}$,

1. $\sum_{n=0}^{\infty} S_{n} y$ converges unconditionally in $X$,

2. $\sum_{n=0}^{k} T^{k} S_{k-n} y$ converges unconditionally in $Y$, uniformly in $k \geq 0$,

3. $\sum_{n=0}^{\infty} T^{k} S_{k+n} y$ converges unconditionally in $Y$, uniformly in $k \geq 0$,

4. $T^{n} S_{n} y \rightarrow y$,

then $T$ is frequently hypercyclic.

Remark 8. Let $\left(x_{n, k}\right)_{n \geq 0, k \in I} \subset X$. A collection of series $\sum_{n=0}^{\infty} x_{n, k}, k \in I$, is said to be unconditionally convergent uniformly in $k \in I$ if for any $\varepsilon>0$, there exists $N \geq 1$ such that for any $k \in I$, any finite set $F \subset[N, \infty[$,

$$
\left\|\sum_{n \in F} x_{k, n}\right\|<\varepsilon
$$

where $\|\cdot\|$ is a F-norm inducing the topology of $X$.

We adapt the Hypercyclicity Criterion and the Frequent Hypercyclicity Criterion to the notion of $\mathcal{A}$-frequent hypercyclicity. 
Theorem 9 ( $\mathcal{A}$-Frequent Hypercyclicity Criterion). Let $X$ be a separable $F$-space, $T \in$ $\mathcal{L}(X)$ and $\mathcal{A}$ a hypercyclicity set. If there exist a dense subset $Y_{0} \subset X, S_{n}: Y_{0} \rightarrow X$, $n \geq 0$, and disjoint sets $A_{k} \in \mathcal{A}, k \geq 1$, such that for each $y \in Y_{0}$,

1. there exists $k_{0} \geq 1$ such that $\sum_{n \in A_{k}} S_{n} y$ converges unconditionally in $X$, uniformly in $k \geq k_{0}$,

2. for any $k_{0} \geq 1$, any $\varepsilon>0$, there exists $k \geq k_{0}$ such that for any finite set $F \subset A_{k}$, any $n \in \bigcup_{l \geq 1} A_{l}, n \notin F$, we have

$$
\left\|\sum_{i \in F} T^{n} S_{i} y\right\| \leq \varepsilon
$$

and such that for any $\delta>0$, there exists $l_{0} \geq 1$ such that for any finite set $F \subset A_{k}$, any $n \in \bigcup_{l \geq l_{0}} A_{l}, n \notin F$, we have

$$
\left\|\sum_{i \in F} T^{n} S_{i} y\right\| \leq \delta
$$

3. $\sup _{n \in A_{k}}\left\|T^{n} S_{n} y-y\right\| \rightarrow 0$ as $k \rightarrow \infty$,

then $T$ is $\mathcal{A}$-frequently hypercyclic.

Proof. Let $\left(y_{l}\right) \subset Y_{0}$ be a dense sequence and $\|\cdot\|$ a $F$-norm inducing the topology of $X$. Without loss of generality, we can suppose that $T$ satisfies the $\mathcal{A}$-Frequent Hypercyclicity Criterion for $Y_{0}=\left\{y_{l}: l \geq 1\right\}$ and for a sequence $\left(A_{k}\right) \subset \mathcal{A}$ of disjoint sets with $A_{k} \subset\left[k, \infty\left[\right.\right.$. Indeed, it suffices to consider a subsequence of the initial sequence $\left(A_{k}\right)$ such that 2 . remains satisfied for any vector $y_{l}$. We then construct recursively an increasing sequence $\left(k_{l}\right)$ such that for any $l \geq 1$,

i. for any finite set $F \subset\left[k_{l}, \infty[\right.$, any $j \leq l$,

$$
\left\|\sum_{n \in F \cap A_{k_{j}}} S_{n} y_{j}\right\| \leq \frac{1}{l 2^{l}},
$$

ii. for any finite set $F \subset A_{k_{l}}$, any $n \in \bigcup_{k \geq 1} A_{k}$,

$$
\left\|\sum_{i \in F \backslash\{n\}} T^{n} S_{i} y_{l}\right\| \leq \frac{1}{2^{l}},
$$

iii. for any $j<l$, any finite set $F \subset A_{k_{j}}$, any $n \in A_{k_{l}}$,

$$
\left\|\sum_{i \in F} T^{n} S_{i} y_{j}\right\| \leq \frac{1}{l 2^{l}}
$$

iv. for any $n \in A_{k_{l}}$,

$$
\left\|T^{n} S_{n} y_{l}-y_{l}\right\| \leq \frac{1}{2^{l}}
$$


Property i. follows from 1., property ii. and property iii. from 2., and property iv. from 3. Moreover, since we suppose $A_{k} \subset[k, \infty[$, property i. implies that for any finite set $F$, for any $j \geq 1$, we have

$$
\left\|\sum_{n \in F \cap A_{k_{j}}} S_{n} y_{j}\right\| \leq \frac{1}{j 2^{j}} .
$$

We write $A:=\bigcup_{l} A_{k_{l}}$ and for any $n \in A$, we let $z_{n}=y_{l}$ if $n \in A_{k_{l}}$. We then consider the vector $x:=\sum_{n \in A} S_{n} z_{n}$. We show that $x$ is well-defined and that $x$ is an $\mathcal{A}$-frequently hypercyclic vector. Let $l \geq 1$ and $F$ be a finite subset of $\left[k_{l}, \infty\right.$ [. We deduce from i. and (3) that

$$
\begin{aligned}
\left\|\sum_{n \in F \cap A} S_{n} z_{n}\right\| & \leq \sum_{j=1}^{l}\left\|\sum_{n \in F \cap A_{k_{j}}} S_{n} y_{j}\right\|+\sum_{j=l+1}^{\infty}\left\|\sum_{n \in F \cap A_{k_{j}}} S_{n} y_{j}\right\| \\
& \leq \sum_{j=1}^{l} \frac{1}{l 2^{l}}+\sum_{j=l+1}^{\infty} \frac{1}{j 2^{j}} \leq \frac{1}{2^{l-1}} \rightarrow 0 .
\end{aligned}
$$

We conclude that the vector $x$ is well-defined. To show that $x$ is $\mathcal{A}$-frequently hypercyclic, it is sufficient to prove that there exists a sequence $C_{l}$ tending to 0 such that for any $l \geq 1$, any $n \in A_{k_{l}}$,

$$
\left\|T^{n} x-y_{l}\right\| \leq C_{l}
$$

Let $l \geq 1$ and $n \in A_{k_{l}}$. We decompose $T^{n} x-y_{l}$ as follows:

$$
T^{n} x-y_{l}=\sum_{\substack{i \in A \\ i<n}} T^{n} S_{i} z_{i}+\sum_{\substack{i \in A \\ i>n}} T^{n} S_{i} z_{i}+T^{n} S_{n} y_{l}-y_{l} .
$$

We already know by iv. that $\left\|T^{n} S_{n} y_{l}-y_{l}\right\| \leq \frac{1}{2^{l}}$. Let $m>n$. We also have

$$
\sum_{\substack{i \in A \\ n<i \leq m}} T^{n} S_{i} z_{i}=\sum_{j=1}^{\infty} \sum_{\substack{i \in A_{k_{j}} \\ n<i \leq m}} T^{n} S_{i} y_{j}=\sum_{j=1}^{\infty} \sum_{i \in F_{k_{j}}} T^{n} S_{i} y_{j}
$$

where $\left.\left.F_{k_{j}}=A_{k_{j}} \cap\right] n, m\right]$. We know by ii. that for any $j \geq 1$,

$$
\left\|\sum_{i \in F_{k_{j}}} T^{n} S_{i} y_{j}\right\| \leq \frac{1}{2^{j}}
$$

and by iii. that for any $j<l$,

$$
\left\|\sum_{i \in F_{k_{j}}} T^{n} S_{i} y_{j}\right\| \leq \frac{1}{l 2^{l}}
$$

We deduce that

$$
\left\|\sum_{\substack{i \in A \\ n<i \leq m}} T^{n} S_{i} z_{i}\right\| \leq \frac{1}{2^{l-2}} \quad \text { and thus } \quad\left\|\sum_{\substack{i \in A \\ i>n}} T^{n} S_{i} z_{i}\right\| \leq \frac{1}{2^{l-2}}
$$


Similarly, we get, with $F_{k_{j}}=A_{k_{j}} \cap[0, n[$,

$$
\left\|\sum_{\substack{i \in A \\ i<n}} T^{n} S_{i} z_{i}\right\|=\left\|\sum_{j=1}^{\infty} \sum_{i \in F_{k_{j}}} T^{n} S_{i} y_{j}\right\| \leq \frac{1}{2^{l-2}} .
$$

We conclude that for any $l \geq 1$, any $n \in A_{k_{l}}$,

$$
\left\|T^{n} x-y_{l}\right\| \leq \frac{1}{2^{l-2}}+\frac{1}{2^{l-2}}+\frac{1}{2^{l}}
$$

which concludes the proof.

We now compare the $\mathcal{A}$-Frequent Hypercyclicity Criterion with the Hypercyclicity Criterion and the Frequent Hypercyclicity Criterion.

Theorem 10. Let $X$ be a separable $F$-space and $T \in \mathcal{L}(X)$. If $T$ satisfies the Hypercyclicity Criterion, then $T$ satisfies the $\mathcal{A}_{\infty}$-Frequent Hypercyclicity Criterion.

Proof. Suppose that $T$ satisfies the Hypercyclicity Criterion for $\left(n_{k}\right),\left(S_{n_{k}}\right), X_{0}$ and $Y_{0}$. Since $X_{0}$ is dense, we can suppose without loss of generality that $S_{n_{k}}\left(Y_{0}\right) \subset X_{0}$ for any $k \geq 1$. Let $\left(y_{j}\right)$ be a dense sequence in $Y_{0}$. There exists a subsequence $\left(m_{k}\right) \subset\left(n_{k}\right)$ such that for any $j, k<l$, we have

$$
\left\|S_{m_{l}} y_{j}\right\| \leq \frac{1}{l^{2}},\left\|T^{m_{k}} S_{m_{l}} y_{j}\right\| \leq \frac{1}{l^{2}}, \quad\left\|T^{m_{l}} S_{m_{k}} y_{j}\right\| \leq \frac{1}{l^{2}} \quad \text { and } \quad\left\|T^{m_{l}} S_{m_{l}} y_{j}-y_{j}\right\| \leq \frac{1}{l^{2}} .
$$

Therefore, one has that $T$ satisfies the $\mathcal{A}$-Frequent Hypercyclicity Criterion for $A_{k}=$ $\left\{m_{p_{k}^{j}}: j \in \mathbb{N}\right\}, k \in \mathbb{N}$, where $p_{1}=2<p_{2}=3<\ldots$ is the increasing enumeration of the prime numbers, and $Y_{0}=\left\{y_{j}: j \in \mathbb{N}\right\}$.

Theorem 11. Let $X$ be a separable F-space, $T \in \mathcal{L}(X)$ and $\mathcal{A}$ a hypercyclicity set. If $T$ satisfies the Frequent Hypercyclicity Criterion, then $T$ satisfies the $\mathcal{A}$-Frequent $H y$ percyclicity Criterion for any sequence $\left(A_{k}\right)_{k \geq 1} \in \mathcal{A}^{\mathbb{N}}$ of disjoint sets such that for any $j \in A_{k}$, any $j^{\prime} \in A_{k^{\prime}}, j \neq j^{\prime}$, we have $\left|j^{\prime}-j\right| \geq \max \left\{k, k^{\prime}\right\}$.

Proof. Suppose that $T$ satisfies the Frequent Hypercyclicity Criterion for $Y_{0}$ and $\left(S_{n}\right)$. Let $\varepsilon>0$ and $y \in Y_{0}$. We then know that there exists $N \geq 1$ such that for any finite set $F \subset[N, \infty[$, we have

- $\left\|\sum_{n \in F} S_{n} y\right\| \leq \varepsilon$,

- for any $k \geq 0,\left\|\sum_{n \in F \cap[0, k]} T^{k} S_{k-n} y\right\| \leq \varepsilon$,

- for any $k \geq 0,\left\|\sum_{n \in F} T^{k} S_{k+n} y\right\| \leq \varepsilon$,

- for any $n \geq N,\left\|T^{n} S_{n} y-y\right\| \leq \varepsilon$. 
In particular, we deduce that for any $k \geq 0$, any finite subset $F \subset \mathbb{Z}_{+}$, if $d(F, k) \geq N$, then

$$
\left\|\sum_{n \in F} T^{k} S_{n} y\right\| \leq\left\|\sum_{n \in F \cap[0, k[} T^{k} S_{k-(k-n)} y\right\|+\left\|\sum_{n \in F \cap] k, \infty[} T^{k} S_{k+(n-k)} y\right\| \leq 2 \varepsilon .
$$

Let $\left(A_{k}\right) \subset \mathcal{A}$ be a sequence of disjoint sets such that for any $j \in A_{k}$, any $j^{\prime} \in A_{k^{\prime}}, j \neq j^{\prime}$, we have $\left|j^{\prime}-j\right| \geq \max \left\{k, k^{\prime}\right\}$. We deduce that the operator $T$ satisfies the assertions 2 . and 3 . of the $\mathcal{A}$-Frequent Hypercyclicity Criterion for the sequence $\left(A_{k}\right)$. It is obvious that $T$ satisfies the assertion 1 . of the $\mathcal{A}$-Frequent Hypercyclicity Criterion and since for any $N \geq 1$, there exists $k_{0}$ such that for any $k \geq k_{0}, A_{k} \cap[0, N]=\emptyset$, we conclude that $T$ also satisfies the assertion 4 . of the $\mathcal{A}$-Frequent Hypercyclicity Criterion for the sequence $\left(A_{k}\right)$.

In particular, we deduce the following result from Theorem 11 .

Corollary 12. Let $X$ be a separable $F$-space and $T \in \mathcal{L}(X)$. If $T$ satisfies the Frequent Hypercyclicity Criterion, then $T$ is $\mathcal{A}$-frequently hypercyclic for any hypercyclicity set $\mathcal{A}$.

\subsection{Characterization of $\mathcal{A}$-frequently hypercyclic weighted shifts}

Weighted shifts are one of the most important family of operators in linear dynamics. The goal of this section consists in showing how we can apply the $\mathcal{A}$ - Hypercyclicity Criterion in order to characterize the $\mathcal{A}$-frequently hypercyclic weighted shifts on $\ell^{p}\left(\mathbb{Z}_{+}\right)$ $(1 \leq p<\infty)$ or on $c_{0}\left(\mathbb{Z}_{+}\right)$.

Theorem 13. Let $\mathcal{A}$ be a hypercyclicity set and $B_{w}$ a weighted shift on $X$ where $X=$ $\ell^{p}\left(\mathbb{Z}_{+}\right)(1 \leq p<\infty)$ or $c_{0}\left(\mathbb{Z}_{+}\right)$. The following assertions are equivalent:

1) $B_{w}$ is $\mathcal{A}$-frequently hypercyclic,

2) $B_{w}$ satisfies the $\mathcal{A}$-Frequent Hypercyclicity Criterion,

3) there is a sequence $\left(A_{k}\right)_{k \geq 1} \subset \mathcal{A}$ of disjoints sets such that

i. for any $j \in A_{k}$, any $j^{\prime} \in A_{k^{\prime}}, j \neq j^{\prime}$, we have $\left|j^{\prime}-j\right| \geq \max \left\{k, k^{\prime}\right\}$.

ii. for any $k^{\prime} \geq 0$, any $k>k^{\prime}$,

$$
\sum_{n \in A_{k}+k^{\prime}} \frac{e_{n}}{\prod_{\nu=1}^{n} w_{\nu}} \in X \quad \text { and } \quad \sum_{n \in A_{k}+k^{\prime}} \frac{e_{n}}{\prod_{\nu=1}^{n} w_{\nu}} \stackrel{k \rightarrow \infty}{\longrightarrow} 0
$$

iii. there exists a family $\left(C_{k, l}\right)_{k, l \geq 1}$ such that for any $k^{\prime} \geq 0$, any $k>k^{\prime}$, any $l \geq 1$,

$$
\sup _{j \in A_{l}}\left\|\sum_{n \in A_{k}-j} \frac{e_{n+k^{\prime}}}{\prod_{\nu=1}^{n} w_{\nu+k^{\prime}}}\right\| \leq C_{k, l}
$$

and such that $\sup _{l} C_{k, l}$ converges to 0 when $k \rightarrow \infty$ and, for any $k \geq 0, C_{k, l}$ converges to 0 when $l \rightarrow \infty$.

Remark 14. In the statement of this theorem and the rest of this section, for any $A \subset \mathbb{Z}_{+}$, any $j \in \mathbb{Z}_{+}$, we denote by $\sum_{n \in A-j}$ the series $\sum_{n \in(A-j) \cap \mathbb{N}}$ and by $\sum_{n \in j-A}$ the series $\sum_{n \in(j-A) \cap \mathbb{N}}$. We also suppose by convention that $\prod_{\nu=1}^{0} w_{\nu}=1$. 
Proof. 3) $\Rightarrow 2)$. Let $S\left(\left(x_{n}\right)_{n \geq 0}\right)=\left(0, \frac{x_{0}}{w_{1}}, \frac{x_{1}}{w_{2}}, \ldots\right)$. We show that $B_{w}$ satisfies the $\mathcal{A}$ Frequent Hypercyclicity Criterion for the set $Y_{0}=\operatorname{span}\left\{e_{k}: k \geq 0\right\}$, the maps $\left(S^{n}\right)_{n \geq 0}$ and the sets $A_{k}$ given by the assertion 3). We notice that for any $x \in Y_{0}$, any $n \geq 1$, any finite set $F \subset \mathbb{Z}_{+}$, we have

$$
\sum_{i \in F \backslash\{n\}} B_{w}^{n} S^{i} x=\sum_{\substack{i \in F \\ i<n}} B_{w}^{n-i} x+\sum_{\substack{i \in F \\ i>n}} S^{i-n} x=\sum_{i \in n-F} B_{w}^{i} x+\sum_{i \in F-n} S^{i} x .
$$

We prove that $B_{w}$ satisfies each assumption of the $\mathcal{A}$-Frequent Hypercyclicity Criterion for any vector $e_{k^{\prime}}$ :

1. Let $k^{\prime} \geq 0$. For any $k>k^{\prime}$, we deduce from ii. that

$$
\sum_{n \in A_{k}} S^{n} e_{k^{\prime}}=\sum_{n \in A_{k}} \frac{e_{k^{\prime}+n}}{\prod_{\nu=1}^{n} w_{\nu+k^{\prime}}}=\prod_{\nu=1}^{k^{\prime}} w_{\nu} \cdot \sum_{n \in A_{k}+k^{\prime}} \frac{e_{n}}{\prod_{\nu=1}^{n} w_{\nu}} \in X .
$$

In particular, each series $\sum_{n \in A_{k}} S^{n} e_{k^{\prime}}$ converges unconditionally and since $\sum_{n \in A_{k}} S^{n} e_{k^{\prime}}$ tends to 0 as $k \rightarrow \infty$ by ii., we deduce that $\sum_{n \in A_{k}} S^{n} e_{k^{\prime}}$ converges unconditionally, uniformly in $k>k^{\prime}$.

2. Let $k^{\prime} \geq 0, k_{0} \geq 0$ and $\varepsilon>0$. For any $k>k^{\prime}$, we deduce from i. that for any $j \in \bigcup_{l} A_{l}$,

$$
\sum_{n \in j-A_{k}} B_{w}^{n} e_{k^{\prime}}=0
$$

and if $j \in A_{l}$, we deduce from iii. that

$$
\left\|\sum_{n \in A_{k}-j} S^{n} e_{k^{\prime}}\right\| \leq C_{k, l}
$$

Since $\sup _{l} C_{k, l} \rightarrow 0$ when $k \rightarrow \infty$, there exists $k>\max \left\{k^{\prime}, k_{0}\right\}$ such that we have for any $j \in \bigcup_{l} A_{l},\left\|\sum_{n \in A_{k}-j} S^{n} e_{k^{\prime}}\right\| \leq \varepsilon$. On the other hand, since $C_{k, l}$ converges to 0 when $l \rightarrow 0$, for any $\delta>0$, there exists $l_{0} \geq 1$ such that for any $l \geq l_{0}$, we have $C_{k, l} \leq \delta$. We conclude by using (4).

3. Obvious.

2) $\Rightarrow$ 1) follows from Theorem 9

$1) \Rightarrow 3$ ). Let $x$ be an $\mathcal{A}$-frequently hypercyclic vector for $B_{w}$. For any $k \geq 1$, any $C_{k}>0$, any $\varepsilon_{k}>0$, there exists $A_{k} \in \mathcal{A}$ such that for any $n \in A_{k}$,

$$
\left\|B_{w}^{n} x-\left(C_{k}+\varepsilon_{k}\right) \sum_{k^{\prime}=0}^{k} e_{k^{\prime}}\right\|<\varepsilon_{k} .
$$

We will fix $C_{k}$ and $\varepsilon_{k}$ later but we already suppose that $\left(C_{k}\right)$ is an increasing sequence tending to $\infty$ and $\left(\varepsilon_{k}\right)$ is a decreasing sequence tending to 0 . We first notice that for any $n \in A_{k}$, any $k^{\prime}>k$, we have

$$
\left|\prod_{\nu=1}^{n} w_{\nu+k^{\prime}} x_{n+k^{\prime}}\right|<\varepsilon_{k}
$$


and for any $0 \leq k^{\prime} \leq k$, we have

$$
\left|\prod_{\nu=1}^{n} w_{\nu+k^{\prime}} x_{n+k^{\prime}}-\left(C_{k}+\varepsilon_{k}\right)\right|<\varepsilon_{k}
$$

and thus

$$
C_{k}<\left|\prod_{\nu=1}^{n} w_{\nu+k^{\prime}} x_{n+k^{\prime}}\right|<C_{k}+2 \varepsilon_{k} .
$$

We show that the sequence $\left(A_{k}\right)_{k \geq 1}$ satisfies i., ii. and iii. for a good choice of $\left(C_{k}\right)$ and $\left(\varepsilon_{k}\right)$ :

1. We show that the sets $\left(A_{k}\right)$ are disjoints and that for any $j \in A_{k}$, any $j^{\prime} \in A_{k^{\prime}}$, $j \neq j^{\prime}$, we have $\left|j^{\prime}-j\right|>\max \left\{k, k^{\prime}\right\}$.

Let $1 \leq k^{\prime}<k$ and $j \in A_{k}$. We have to show that for any $0 \leq n \leq k$, we have $j+n \notin A_{k^{\prime}}$ and $j-n \notin A_{k^{\prime}}$. Let $0 \leq n \leq k$. We know by (6) that $\left|\prod_{\nu=1}^{j} w_{\nu+k} x_{j+k}\right|>C_{k}$. We deduce that

$$
\left|\prod_{\nu=1}^{j+n} w_{\nu+k-n} x_{j+k}\right|>C_{k} \min _{0 \leq n^{\prime} \leq k} \min _{\nu \leq k}\left|w_{\nu}\right|^{n^{\prime}}
$$

and

$$
\left|\prod_{\nu=1}^{j-n} w_{\nu+k+n} x_{j+k}\right|>\frac{C_{k}}{\max _{0 \leq n^{\prime} \leq k} \max _{k \leq \nu \leq 2 k}\left|w_{\nu}\right|^{n^{\prime}}} .
$$

On the other hand, we also know by (5) and (6) that for any $m \in A_{k^{\prime}}$, any $l \geq 0$,

$$
\left|\prod_{\nu=1}^{m} w_{\nu+l} x_{m+l}\right|<C_{k^{\prime}}+2 \varepsilon_{k^{\prime}}
$$

In particular, if $j+n \in A_{k^{\prime}}$, we would have, for $l=k-n$,

$$
\left|\prod_{\nu=1}^{j+n} w_{\nu+k-n} x_{j+k}\right|<C_{k^{\prime}}+2 \varepsilon_{k^{\prime}} \leq C_{k-1}+2 \varepsilon_{1} .
$$

We then deduce from $(7)$ and $(10)$ that if we suppose

$$
C_{k}>\frac{C_{k-1}+2 \varepsilon_{1}}{\min _{0 \leq n^{\prime} \leq k} \min _{\nu \leq k}\left|w_{\nu}\right|^{n^{\prime}}},
$$

then for any $k^{\prime}<k$, any $0 \leq n \leq k$, we have $j+n \notin A_{k^{\prime}}$. On the other hand, if we suppose that $j-n \in A_{k^{\prime}}$, then we would have by (9) for $l=k+n$,

$$
\left|\prod_{\nu=1}^{j-n} w_{\nu+k+n} x_{j+k}\right|<C_{k^{\prime}}+2 \varepsilon_{k^{\prime}} \leq C_{k-1}+2 \varepsilon_{1} .
$$

Hence, if we suppose

$$
C_{k}>\left(C_{k-1}+2 \varepsilon_{1}\right) \max _{0 \leq n^{\prime}<k} \max _{k \leq \nu \leq 2 k}\left|w_{\nu}\right|^{n^{\prime}}
$$


then, by (8) and (12), we deduce that for any $k^{\prime}<k$, any $0 \leq n \leq k$, we have $j-n \notin A_{k^{\prime}}$ and thus $d\left(A_{k^{\prime}}, A_{k}\right)>k$.

If $k=k^{\prime}$, it suffices to show that for any $j \in A_{k}$, any $1 \leq n \leq k$, we have $j-n \notin A_{k}$. By (5), we know that for any $m \in A_{k}$, any $l>k$,

$$
\left|\prod_{\nu=1}^{m} w_{\nu+l} x_{m+l}\right|<\varepsilon_{k} .
$$

If $j-n \in A_{k}$, we would thus have, for $l=k+n$,

$$
\left|\prod_{\nu=1}^{j-n} w_{\nu+k+n} x_{j+k}\right|<\varepsilon_{k} \leq \varepsilon_{1} .
$$

We deduce from (8) and (14) that if

$$
C_{k}>\varepsilon_{1} \max _{0 \leq n^{\prime} \leq k} \max _{k \leq \nu \leq 2 k}\left|w_{\nu}\right|^{n^{\prime}}
$$

then $j-n \notin A_{k}$ for any $1 \leq n \leq k$.

The property i. is thus satisfied if we choose $\left(C_{k}\right)$ such that Conditions (11), (13) and (15) are satisfied. For the sequence $\left(\varepsilon_{k}\right)$, we can consider any decreasing sequence tending to 0 .

2. Let $k^{\prime} \geq 0$. We know by (6) that for any $k>k^{\prime}$, any $n \in A_{k}$,

$$
\frac{1}{\prod_{\nu=1}^{n}\left|w_{\nu+k^{\prime}}\right|}<\frac{\left|x_{n+k^{\prime}}\right|}{C_{k}}
$$

Hence, we have

$$
\begin{aligned}
\left\|\sum_{n \in A_{k}+k^{\prime}} \frac{e_{n}}{\prod_{\nu=1}^{n} w_{\nu}}\right\| & =\left\|\sum_{n \in A_{k}} \frac{e_{n+k^{\prime}}}{\prod_{\nu=1}^{n+k^{\prime}} w_{\nu}}\right\| \\
& =\frac{1}{\prod_{\nu=1}^{k^{\prime}}\left|w_{\nu}\right|}\left\|\sum_{n \in A_{k}} \frac{e_{n+k^{\prime}}}{\prod_{\nu=1}^{n}\left|w_{\nu+k^{\prime}}\right|}\right\| \\
& \leq \frac{\|x\|}{C_{k} \prod_{\nu=1}^{k^{\prime}}\left|w_{\nu}\right|}<\infty
\end{aligned}
$$

and since $C_{k} \rightarrow \infty$, we deduce that

$$
\sum_{n \in A_{k}+k^{\prime}} \frac{e_{n}}{\prod_{\nu=1}^{n} w_{\nu}} \stackrel{k \rightarrow \infty}{\longrightarrow} 0
$$

3. We show that for any $k^{\prime} \geq 0$, any $k>k^{\prime}$, any $l \geq 1$, any $j \in A_{l}$, we have

$$
\left\|\sum_{n \in A_{k}-j} \frac{C_{k}}{\prod_{\nu=1}^{n} w_{\nu+k^{\prime}}} e_{n+k^{\prime}}\right\|<\varepsilon_{l} .
$$


Let $k>k^{\prime} \geq 0, l \geq 1$ and $j \in A_{l}$. We have, by definition of $A_{l}$,

$$
\begin{aligned}
\varepsilon_{l} & >\left\|B_{w}^{j} x-\left(C_{l}+\varepsilon_{l}\right) \sum_{l^{\prime}=0}^{l} e_{l^{\prime}}\right\| \geq\left\|\sum_{n=l+1}^{\infty}\left(\prod_{\nu=1}^{j} w_{\nu+n}\right) x_{j+n} e_{n}\right\| \\
& \geq\left\|\sum_{n=l+1}^{\infty}\left(\prod_{\nu=1}^{j} w_{\nu+n+k^{\prime}}\right) x_{j+n+k^{\prime}} e_{n+k^{\prime}}\right\| \\
& \geq\left\|\sum_{n \in A_{k}-j}\left(\prod_{\nu=1}^{j} w_{\nu+n+k^{\prime}}\right) x_{j+n+k^{\prime}} e_{n+k^{\prime}}\right\| \text { by } 1 . \\
& =\left\|\sum_{n \in A_{k}}\left(\prod_{n>j}^{j} w_{\nu+n-j+k^{\prime}}\right) x_{n+k^{\prime}} e_{n-j+k^{\prime}}\right\| \\
& =\left\|\sum_{n \in A_{k}}\left(\frac{\prod_{\nu=1}^{n} w_{\nu+k^{\prime}}}{\prod_{\nu=1}^{n-j} w_{\nu+k^{\prime}}}\right) x_{n+k^{\prime}} e_{n-j+k^{\prime}}\right\| \\
& \geq\left\|\sum_{n \in A_{k}} \frac{C_{k}}{\prod_{\nu=1}^{n-j} w_{\nu+k^{\prime}}} e_{n-j+k^{\prime}}\right\| \text { by }(6) \\
& =\left\|\sum_{n \in A_{k}-j} \frac{C_{k}}{\prod_{\nu=1}^{n} w_{\nu+k^{\prime}}} e_{n+k^{\prime}}\right\| .
\end{aligned}
$$

We deduce that

$$
\left\|\sum_{n \in A_{k}-j} \frac{e_{n+k^{\prime}}}{\prod_{\nu=1}^{n} w_{\nu+k^{\prime}}}\right\| \leq \frac{\varepsilon_{l}}{C_{k}}
$$

and since

$$
\sup _{l} \frac{\varepsilon_{l}}{C_{k}} \leq \frac{\varepsilon_{1}}{C_{k}} \underset{k \rightarrow \infty}{\longrightarrow} 0 \text { and } \quad \frac{\varepsilon_{l}}{C_{k}} \underset{l \rightarrow \infty}{\longrightarrow} 0
$$

we obtain the desired result.

An important result of Bayart and Ruzsa [6] about frequently hypercyclic weighted shifts on $\ell^{p}$ is that a weighted shift $B_{w}$ on $\ell^{p}$ is frequently hypercyclic if and only if $B_{w}$ is chaotic and thus if and only if

$$
\sum_{n=1}^{\infty} \frac{1}{\prod_{\nu=1}^{n}\left|w_{\nu}\right|^{p}}<\infty .
$$

However, we know that this equivalence is false for weighted shifts on $c_{0}$. Indeed, Bayart and Grivaux [3] have exhibited a frequently hypercyclic weighted shift on $c_{0}$ that is neither chaotic nor mixing. The characterization of frequently hypercyclic weighted shifts that we obtain in term of weights is not satisfactory in the case of the spaces $\ell^{p}$ if we compare it with the characterization obtained by Bayart and Ruzsa. However, in the case of frequently hypercyclic weighted shifts on $c_{0}$, the obtained characterization is similar to the characterization given in [6].

Thanks to the counterexample of Bayart and Grivaux, we also know that the Frequent Hypercyclicity Criterion does not characterize frequently hypercyclic operators, because 
if $T$ satisfies the Frequent Hypercyclicity Criterion, then $T$ is mixing and chaotic. However, the characterization given by Theorem 13 tells us that each frequently hypercyclic weighted shift on $c_{0}$ satisfies the $\mathcal{A}$-Frequent Hypercyclicity Criterion when $\mathcal{A}$ is the family of positive lower density sets. A direct consequence is the existence of operators that are neither mixing nor chaotic and that satisfy the $\mathcal{A}$-Frequent Hypercyclicity Criterion when $\mathcal{A}$ is the family of positive lower density sets. One can thus wonder if the $\mathcal{A}$-Frequent Hypercyclicity Criterion characterizes the frequently hypercyclic operators when $\mathcal{A}$ is the set of positive lower density sets.

\section{Banach densities and reiterative hypercyclicity}

The purpose of this section is to analyze which kind of properties of density can have the sets $N(x, U)$ and classify the hypercyclic operators accordingly to these properties. We first remark that there does not exist $\mathcal{A}$-frequently hypercyclic operators if $\mathcal{A}$ is the family of sets with positive lower Banach density or if $\mathcal{A}$ is the family of sets with upper Banach density equal to 1.

Proposition 15. Let $X \neq\{0\}$ be a F-space. If $\mathcal{A}$ is the family of sets with positive lower Banach density, then $X$ does not support an $\mathcal{A}$-frequently hypercyclic operator.

Proof. Assume towards a contradiction that there exists an $\mathcal{A}$-frequently hypercyclic operator $T$ on $X$. Let $x \in X$ be an $\mathcal{A}$-frequently hypercyclic vector for $T$.

Take $U, V$ non-empty open sets such that $x \in U, 0 \in V$ and $U \cap V=\emptyset$. If we denote the maximum gap of $N(x, U)$ as $m$, then by continuity there exists $W$ a neighbourhood of zero such that $T^{j}(W) \subset V, j=0,1, \ldots, m$. Let $n$ such that $T^{n} x \in W$. We deduce that $T^{n+j} x \in V$ for any $0 \leq j \leq m$ and therefore $\{n, n+1, \ldots, n+m\} \notin N(x, U)$ which is a contradiction since this implies that there are gaps in $N(x, U)$ with length greater than $m$.

Proposition 16. Let $X \neq\{0\}$ be a $F$-space. If $\mathcal{A}$ is the family of sets with upper Banach density equals to 1 , then $X$ does not support an $\mathcal{A}$-frequently hypercyclic operator.

Proof. Assume towards a contradiction that there exists an $\mathcal{A}$-frequently hypercyclic operator $T$ on $X$. Let $x \in X$ be an $\mathcal{A}$-frequently hypercyclic operator. This implies that for every non-empty open set $U$, there exists $n$ such that $n, n+1 \in N(x, U)$ and thus for every non-empty open set $U$, we have

$$
T(U) \cap U \neq \emptyset
$$

Let $z \neq y \in X$ such that $T z=y$. Since $X$ is metrizable, there exists open neighborhoods $V_{y}, V_{z}$ of $y$ and $z$ respectively, such that $V_{y} \cap V_{z}=\emptyset$. On the other hand, by continuity of $T$ there exists an open neighborhood of $z$ denoted $\tilde{V}_{z}$, such that $T\left(\tilde{V}_{z}\right) \subset V_{y}$. Let $\hat{V}_{z}=V_{z} \cap \tilde{V}_{z}$. We get that $\hat{V}_{z}$ is a non-empty open set and since $T\left(\hat{V}_{z}\right) \subset V_{y}$, we conclude that $T\left(\hat{V}_{z}\right) \cap \hat{V}_{z}=\emptyset$. This is a contradiction with condition (17).

We deduce from Proposition 15. Proposition 16 and (1) that there are only three possibilities:

1. $T$ is frequently hypercyclic i.e. $\underline{d}(N(x, U))>0$;

2. $T$ is $\mathfrak{U}$-frequently hypercyclic i.e. $\bar{d}(N(x, U))>0$; 
3. $T$ is reiteratively hypercyclic i.e. $\overline{B d}(N(x, U))>0$.

Obviously, for any operator $T$, we have

frequently hypercyclic $\Rightarrow \mathfrak{U}$-frequently hypercyclic $\Rightarrow$ reiteratively hypercyclic.

In the case of weighted shifts on $\ell^{p}$, we have even an equivalence between frequent hypercyclicity and $\mathfrak{U}$-frequent hypercyclicity [6. Nevertheless, this equivalence is false in general and in particular for weighted shifts on $c_{0}$ [6]. Thanks to weighted shifts on $c_{0}$, we can also show that there is not, in general, an equivalence between reiterative hypercyclicity and $\mathfrak{U}$-frequent hypercyclicity.

Theorem 17. There exists some reiteratively hypercyclic weighted shift on $c_{0}\left(\mathbb{Z}_{+}\right)$that is not $\mathfrak{U}$-frequently hypercyclic.

Proof. Let $\left.S:=\bigcup_{j, l \geq 1}\right] l 10^{j}-j, l 10^{j}+j[$. We consider the weighted sequence $w$ given by

$$
w_{k}=\left\{\begin{array}{cl}
2 & \text { if } k \in S \\
\prod_{\nu=1}^{k-1} w_{\nu}^{-1} & \text { if } k \in(S+1) \backslash S \\
1 & \text { otherwise. }
\end{array}\right.
$$

In particular, we deduce from the definition that $\prod_{\nu=1}^{n} w_{\nu}=1$ if and only if $n \notin S$, and $\prod_{\nu=1}^{n} w_{\nu} \geq 2^{j}$ if and only if $\left.] n-j, n\right] \subset S$.

We first show that $B_{w}$ is reiteratively hypercyclic by using Theorem 13 . To this end, we have to construct a sequence $\left(A_{k}\right)_{k \geq 1}$ of disjoints sets with positive upper Banach density such that

i. for any $j \in A_{k}$, any $j^{\prime} \in A_{k^{\prime}}, j \neq j^{\prime}$, we have $\left|j^{\prime}-j\right| \geq \max \left\{k, k^{\prime}\right\}$.

ii. for any $k^{\prime} \geq 0$, any $k>k^{\prime}$,

$$
\prod_{\nu=1}^{n} w_{\nu} \stackrel{n \in A_{k}+k^{\prime}}{\longrightarrow} \infty \quad \text { and } \quad \sup _{n \in A_{k}+k^{\prime}} \frac{1}{\prod_{\nu=1}^{n} w_{\nu}} \stackrel{k \rightarrow \infty}{\longrightarrow} 0
$$

iii. there exists a family $\left(C_{k, l}\right)_{k, l \geq 1}$ such that for any $k^{\prime} \geq 0$, any $k>k^{\prime}$, any $l \geq 1$,

$$
\sup _{j \in A_{l}} \sup _{n \in A_{k}-j} \frac{1}{\prod_{\nu=1}^{n} w_{\nu+k^{\prime}}} \leq C_{k, l}
$$

and such that $\sup _{l} C_{k, l}$ converges to 0 when $k \rightarrow \infty$ and, for any $k \geq 0, C_{k, l}$ converges to 0 when $l \rightarrow \infty$.

Let $\phi: \mathbb{N} \rightarrow \mathbb{N}$ such that for any $k \geq 1$, we have $\#\{j \geq 1: \phi(j)=k\}=\infty$. We construct a sequence of sets $\left(F_{j}\right)_{j \geq 1}$ such that if $A_{k}:=\bigcup_{\phi(j)=k} F_{j}$, then the sequence $\left(A_{k}\right)_{k \geq 1}$ satisfies the desired properties.

Let $F_{0}=\{0\}$. If we have already constructed $F_{1}, \cdots, F_{j}$ and $\phi(j+1)=k$, then we let $F_{j+1}=\left\{10^{j_{0}}+10^{2 k} l: l \in\left[0, l_{0}[\}\right.\right.$ where $j_{0}$ and $l_{0}$ are positive integers satisfying

1) $l_{0} \geq j+1$ 
2) $10^{j_{0}} \geq k+\max _{1 \leq n \leq j} \phi(n)+\max \left(\bigcup_{n=0}^{j} F_{n}\right)$;

3) $j_{0} \geq j+1$ and $j_{0}-k>10^{2 k} l_{0}$;

4) $j_{0}>\max \left(\bigcup_{n=0}^{j} F_{n}\right)+\max _{1 \leq n \leq j} \phi(n)+2 k$.

Let $A_{k}:=\bigcup_{\phi(j)=k} F_{j}$. We first remark that for any real number $s \geq 1$, any $k \geq 1$,

$$
\begin{aligned}
\alpha_{k}^{s}:=\limsup _{l \rightarrow \infty}\left|A_{k} \cap[l+1, l+s]\right| & \geq \limsup _{j \rightarrow \infty, \phi(j)=k}\left|F_{j} \cap\left[\min F_{j}+1, \min F_{j}+s\right]\right| \\
& \left.\geq \limsup _{j \rightarrow \infty, \phi(j)=k} \min \left\{\left\lfloor\frac{s}{10^{2 k}}\right\rfloor, j\right\} \quad \text { by } 1\right) \\
& =\left\lfloor\frac{s}{10^{2 k}}\right\rfloor .
\end{aligned}
$$

We deduce that for any $k \geq 1$

$$
\overline{B d}\left(A_{k}\right):=\lim _{s \rightarrow \infty} \frac{\alpha_{k}^{s}}{s} \geq \lim _{s \rightarrow \infty} \frac{\left\lfloor\frac{s}{10^{2 k}}\right\rfloor}{s}=\frac{1}{10^{2 k}}>0 .
$$

Moreover, the sets $A_{k}$ are disjoint since it follows from 2) that the sets $F_{j}$ are disjoint. In fact, 2) implies that for any $j \in A_{k}$, any $j^{\prime} \in A_{k^{\prime}}, j \neq j^{\prime}$, we have $\left|j^{\prime}-j\right| \geq \max \left\{k, k^{\prime}\right\}$. Indeed if $j \in A_{k}$, there exists $n \geq 1$ such that $j \in F_{n}$ and $\phi(n)=k$, and if $j^{\prime} \in A_{k^{\prime}}$, there exists $n^{\prime} \geq 1$ such that $j^{\prime} \in F_{n^{\prime}}$ and $\phi\left(n^{\prime}\right)=k^{\prime}$. Therefore, if $n=n^{\prime}$, we have $k=k^{\prime}$ and $\left|j-j^{\prime}\right|$ is a non-zero multiple of $10^{2 k} \geq k$. On the other hand, if $n \neq n^{\prime}$, we can assume without loss of generality that $n^{\prime}<n$ and we deduce from 2) that

$$
\left|j-j^{\prime}\right| \geq k+\max _{1 \leq m<n} \phi(m) \geq k+\phi\left(n^{\prime}\right)=k+k^{\prime} \geq \max \left\{k, k^{\prime}\right\} .
$$

It remains to prove that the sets $A_{k}$ satisfy Condition ii. and iii. of Theorem 13 . Condition ii. is satisfied because for any $k \geq 1$, any $j \geq 1$ with $\phi(j)=k$, it follows from 3) that $F_{j} \subset\left[10^{j_{0}}, 10^{j_{0}}+j_{0}-k\right.$ [ for some $j_{0} \geq j$. Therefore, for any $k^{\prime}<k$, we have $F_{j}+k^{\prime} \subset\left[10^{j_{0}}, 10^{j_{0}}+j_{0}\left[\right.\right.$ and thus, by definition of $w$, for any $n \in F_{j}+k^{\prime}$, we have

$$
\prod_{\nu=1}^{n} w_{\nu} \geq 2^{j_{0}} \geq 2^{j} .
$$

Since each $F_{j}$ is finite, we then get, by definition of $A_{k}$, for any $k^{\prime}<k$

$$
\prod_{\nu=1}^{n} w_{\nu} \stackrel{n \in A_{k}+k^{\prime}}{\longrightarrow} \infty
$$

and since $\min \left(\phi^{-1}(\{k\})\right) \rightarrow \infty$ as $k \rightarrow \infty$, we get

$$
\sup _{n \in A_{k}+k^{\prime}} \frac{1}{\prod_{\nu=1}^{n} w_{\nu}} \stackrel{k \rightarrow \infty}{\longrightarrow} 0 .
$$

Finally, for any $k^{\prime} \geq 0$, any $k>k^{\prime}$, any $j \geq 1$ such that $\phi(j)=k$, if $n=m-m^{\prime} \geq 1$ with $m \in F_{j}$ and $m^{\prime} \in F_{j^{\prime}}$, we have two possibilities either $j=j^{\prime}$ or $j>j^{\prime}$. If $j=j^{\prime}$, we deduce from the definition of $F_{j}$ that $n=l 10^{2 k}$ for some $l \geq 1$ and thus

$$
\prod_{\nu=1}^{n} w_{\nu+k^{\prime}}=\frac{\prod_{\nu=1}^{n+k^{\prime}} w_{\nu}}{\prod_{\nu=1}^{k^{\prime}} w_{\nu}} \geq \frac{2^{2 k}}{2^{k^{\prime}}} \geq 2^{k}
$$


On the other hand, if $j>j^{\prime}$, we deduce from 4) that

$$
n \in\left[10^{j_{0}}+2 k+\phi\left(j^{\prime}\right)-j_{0}, 10^{j_{0}}+j_{0}-k[\right.
$$

for some $j_{0} \geq 1$ and thus $n+k^{\prime} \in\left[10^{j_{0}}+2 k+\phi\left(j^{\prime}\right)-j_{0}, 10^{j_{0}}+j_{0}[\right.$. We conclude that

$$
\prod_{\nu=1}^{n} w_{\nu+k^{\prime}}=\frac{\prod_{\nu=1}^{n+k^{\prime}} w_{\nu}}{\prod_{\nu=1}^{k^{\prime}} w_{\nu}} \geq \frac{2^{2 k+\phi\left(j^{\prime}\right)}}{2^{k^{\prime}}} \geq 2^{k+\phi\left(j^{\prime}\right)} .
$$

Let $C_{k, k}:=2^{-k}$ and $C_{k, l}:=2^{-(k+l)}$ if $k \neq l$. We deduce that for any $k^{\prime} \geq 0$, any $k>k^{\prime}$, any $l \geq 1$, we have

$$
\sup _{m^{\prime} \in A_{l}} \max _{n \in A_{k}-m^{\prime}} \frac{1}{\prod_{\nu=1}^{n} w_{\nu+k^{\prime}}} \leq C_{k, l}
$$

and we remark that $\sup _{l} C_{k, l}=2^{-k}$ converges to 0 when $k \rightarrow \infty$ and, for any $k \geq 0$, $C_{k, l}$ converges to 0 when $l \rightarrow \infty$. Condition iii. of Theorem 13 is thus satisfied and we conclude that $B_{w}$ is reiteratively hypercyclic.

We now show that $B_{w}$ is not $\mathfrak{U}$-frequently hypercyclic. Assume that $B_{w}$ is $\mathfrak{U}$-frequently hypercyclic. We then deduce from Theorem 13 that there exists a set $A$ with positive upper density such that

$$
\sum_{n \in A} \frac{e_{n}}{\prod_{\nu=1}^{n} w_{\nu}} \in c_{0}
$$

In other words, we have $\bar{d}(A)>0$ and

$$
\prod_{\nu=1}^{n} w_{\nu} \stackrel{n \in A}{\longrightarrow} \infty
$$

Let $D_{j}:=\left\{n \geq 1: \prod_{\nu=1}^{n} w_{\nu} \geq 2^{j}\right\}$. We remark that $\bar{d}(A) \leq \bar{d}\left(D_{j}\right)$. In order to prove that $B_{w}$ is not $\mathfrak{U}$-frequently hypercyclic, it is thus sufficient to prove that $\bar{d}\left(D_{j}\right) \rightarrow 0$ when $j \rightarrow \infty$. To this end, we will need the following fact.

Fact 1. Let $\left.S:=\bigcup_{j, l \geq 1}\right] l 10^{j}-j, l 10^{j}+j[$. Let $k \geq 1, l \geq 1$ and $n \geq 0$ such that $10^{n-1}<k \leq 10^{n}$. If $m \in\left\{l 10^{k}+\sum_{j=0}^{n} 10^{j}, l 10^{k}-\sum_{j=0}^{n} 10^{j}\right\}$, then

$$
\text { either } m \notin S \text { or } m \in] l_{0} 10^{j_{0}}-j_{0}, l_{0} 10^{j_{0}}+j_{0}\left[\text { for some } j_{0}>k\right. \text {. }
$$

Thanks to Fact 1, we can show that

$$
\left.D_{j}:=\left\{n \geq 1: \prod_{\nu=1}^{n} w_{\nu} \geq 2^{j}\right\} \subseteq \bigcup_{k \geq\left\lceil\frac{j}{30}\right\rceil} \bigcup_{l \geq 1}\right] l 10^{k}-31 k, l 10^{k}+31 k\left[:=E_{j} .\right.
$$

Let $n \notin E_{j}$. We want to show that $n \notin D_{j}$. In other words, we have to show that

$$
] n-j, n] \cap S^{c} \neq \emptyset \text {. }
$$

We first remark that $] n-j, n] \cap] l 10^{k}-k, l 10^{k}+k\left[=\emptyset\right.$ for any $k \geq\left\lceil\frac{j}{30}\right\rceil$ and any $l \geq 1$. Indeed, if $] n-j, n] \cap] l 10^{k}-k, l 10^{k}+k\left[\neq \emptyset\right.$, then $n \geq l 10^{k}-k$ and $n-j<l 10^{k}+k$. Hence,

$$
l 10^{k}-31 k<n=(n-j)+j<l 10^{k}+k+j \leq l 10^{k}+31 k
$$


and thus $n \in E_{j}$, which is a contradiction.

Assume that $] n-j, n] \subset S$ and let

$$
\left.\left.k_{0}:=\max \{k \geq 1:] n-j, n\right] \cap \bigcup_{l \geq 1}\right] l 10^{k}-k, l 10^{k}+k[\neq \emptyset\} .
$$

We deduce from the above reasoning that $k_{0}<\left\lceil\frac{j}{30}\right\rceil$. We consider $\left.\left.m \in\right] n-j, n\right]$ and $l_{0} \geq 1$ such that

$$
m \in] l_{0} 10^{k_{0}}-k_{0}, l_{0} 10^{k_{0}}+k_{0}[\text {. }
$$

Let $n \geq 0$ such that $10^{n-1}<k_{0} \leq 10^{n}, m_{1}:=l_{0} 10^{k_{0}}-\sum_{j=0}^{n} 10^{j}$ and $m_{2}:=l_{0} 10^{k_{0}}+$ $\sum_{j=0}^{n} 10^{j}$. Since $\left.\left.m \in\right] n-j, n\right], m \in\left[m_{1}, m_{2}\right]$ and

$$
m_{2}-m_{1}+1 \leq 3(10)^{n}<30 k_{0} \leq j
$$

we deduce that either $\left.\left.m_{1} \in\right] n-j, n\right]$ or $\left.\left.m_{2} \in\right] n-j, n\right]$. Therefore $m_{1}$ or $m_{2}$ belongs to $S$ and we deduce from Fact 1 that $m_{1}$ or $m_{2}$ belongs to $] l_{1} 10^{k_{1}}-k_{1}, l_{1} 10^{k_{1}}+k_{1}$ [ for some $k_{1}>k_{0}$ and some $l_{1} \geq 1$ which is a contradiction with the definition of $k_{0}$.

If $N \in\left[10^{m}, 10^{m+1}\right.$ [ with $m \geq 1$, we thus deduce that

$$
\begin{aligned}
\frac{\#\left(D_{j} \cap[1, N]\right)}{N} & \leq \frac{\#\left(D_{j} \cap\left[1,10^{m+1}\right]\right)}{10^{m}} \\
& \leq \frac{\#\left(E_{j} \cap\left[1,10^{m+1}\right]\right)}{10^{m}} \leq \frac{\sum_{k=\left\lceil\frac{j}{30}\right\rceil}^{m} 62 k 10^{m+1-k}}{10^{m}} \\
& =\frac{620}{81}\left(\frac{\left(9\left\lceil\frac{j}{30}\right\rceil+1\right) 10^{m+1-\left\lceil\frac{j}{30}\right\rceil}-9 m-10}{10^{m}}\right) \\
& \leq 8\left(9\left\lceil\frac{j}{30}\right\rceil+1\right) 10^{1-\left\lceil\frac{j}{30}\right\rceil} .
\end{aligned}
$$

where the third inequality follows from the fact that for any $\left\lceil\frac{j}{30}\right\rceil \leq k \leq m-1$, there is less than $10^{m+1-k}$ intervals of the form $] l 10^{k}-31 k, l 10^{k}+31 k\left[\right.$ with $l \geq 1$ in $E_{j} \cap\left[1,10^{m+1}\right], 9$ intervals of the form $] l 10^{m}-31 m, l 10^{m}+31 m$ [ and the interval $] 10^{m+1}-31(m+1), 10^{m+1}$. We deduce that $\bar{d}\left(D_{j}\right) \rightarrow 0$ as $j \rightarrow \infty$ and we thus conclude that $B_{w}$ is not $\mathfrak{U}$-frequently hypercyclic.

We finish this proof by giving the proof of Fact 1 .

Proof of Fact 1. We first assume that $m=l 10^{k}+\sum_{j=0}^{n} 10^{j}$. If $m \in S$, then there exists $l_{0} \geq 1$ and $j_{0} \geq 1$ such that $\left.m \in\right] l_{0} 10^{j_{0}}-j_{0}, l_{0} 10^{j_{0}}+j_{0}[$. Therefore, it suffices to prove that $m \notin] l_{1} 10^{j_{1}}-j_{1}, l_{1} 10^{j_{1}}+j_{1}\left[\right.$ for any $1 \leq j_{1} \leq k$, any $l_{1} \geq 1$.

If $n+1 \leq j_{1} \leq k$, then $l 10^{k-j_{1}} 10^{j_{1}} \leq m \leq\left(l 10^{k-j_{1}}+1\right) 10^{j_{1}}$ and we remark that

$$
m \geq l 10^{k}+10^{n} \geq l 10^{k-j_{1}} 10^{j_{1}}+j_{1}
$$

and

$$
m \leq l 10^{k}+10^{j_{1}}-10^{j_{1}-1} \leq\left(l 10^{k-j_{1}}+1\right) 10^{j_{1}}-j_{1} .
$$

Hence $m \notin] l_{1} 10^{j_{1}}-j_{1}, l_{1} 10^{j_{1}}+j_{1}\left[\right.$ for any $n+1 \leq j_{1} \leq k$ and any $l_{1} \geq 1$. On the other hand, if $1 \leq j_{1} \leq n$ then

$$
\left(l 10^{k-j_{1}}+\sum_{j=j_{1}}^{n} 10^{j-j_{1}}\right) 10^{j_{1}} \leq m \leq\left(l 10^{k-j_{1}}+\sum_{j=j_{1}}^{n} 10^{j-j_{1}}+1\right) 10^{j_{1}} .
$$


However,

$$
m \geq l 10^{k}+\sum_{j=j_{1}}^{n} 10^{j}+10^{j_{1}-1} \geq\left(l 10^{k-j_{1}}+\sum_{j=j_{1}}^{n} 10^{j-j_{1}}\right) 10^{j_{1}}+j_{1}
$$

and

$$
m \leq l 10^{k}+\sum_{j=j_{1}}^{n} 10^{j}+10^{j_{1}}-10^{j_{1}-1} \leq\left(l 10^{k-j_{1}}+\sum_{j=j_{1}}^{n} 10^{j-j_{1}}+1\right) 10^{j_{1}}-j_{1} .
$$

So we conclude that $m \notin] l_{1} 10^{j_{1}}-j_{1}, l_{1} 10^{j_{1}}+j_{1}\left[\right.$ for any $1 \leq j_{1} \leq k$ and any $l_{1} \geq 1$.

If we assume that $m=l 10^{k}-\sum_{j=0}^{n} 10^{j}$ and $m \in S$, we can show similarly that $m \notin] l_{1} 10^{j_{1}}-j_{1}, l_{1} 10^{j_{1}}+j_{1}\left[\right.$ for any $1 \leq j_{1} \leq k$ and any $l_{1} \geq 1$. This concludes the proof.

In the case of weighted shifts on $\ell^{p}(\mathbb{Z})$ or $\ell^{p}\left(\mathbb{Z}_{+}\right)$, we can generalize the equivalence obtained by Bayart and Ruzsa [6] by showing that every reiteratively hypercyclic weighted shift on $\ell^{p}(\mathbb{Z})$ or $\ell^{p}\left(\mathbb{Z}_{+}\right)$is frequently hypercyclic. The proof of the characterization obtained by Bayart and Ruzsa is based on the fact that if $A$ is a set with upper density $\bar{d}(A)=\delta>0$ and $\delta_{k}=\bar{d}(A \cap(A-k))$, then the set $F=\left\{k: \delta_{k}>(1-\varepsilon) \delta^{2}\right\}$ is syndetic [6, Theorem 8]. We remark that this result can be extended to sets with positive upper Banach density thanks to following two Theorems.

Theorem 18. (Furstenberg Correspondence principle [17, Theorem 0.2 ]) Given a subset $A \subset \mathbb{Z}$ of positive upper Banach density, there exists a measure-preserving system $(X, \mathfrak{B}, \mu, T)$ and a set $E \in \mathfrak{B}$ such that $\mu(E)=\overline{B d}(A)$ and

$$
\overline{B d}\left(A \cap\left(A-n_{1}\right) \cap \cdots \cap\left(A-n_{k}\right)\right) \geq \mu\left(E \cap T^{-n_{1}} E \cap \cdots \cap T^{-n_{k}} E\right)
$$

for any integer $n_{1}, \ldots, n_{k}$.

Theorem 19. ([7, Theorem 3.1]) For every measure-preserving system $(X, \mathfrak{B}, \mu, T)$, any $\varepsilon>0$ and $A \in \mathfrak{B}$ the set $\left\{n \in \mathbb{Z}: \mu\left(A \cap T^{-n} A\right)>\mu(A)^{2}-\varepsilon\right\}$ is syndetic.

We directly deduce from these theorems the following generalization of result of Bayart and Rusza.

Theorem 20. Let $A \subset \mathbb{Z}$ be a set with positive upper Banach density equal to $\delta$ and $\varepsilon \in] 0,1\left[\right.$. For any $k \in \mathbb{Z}$, let $B_{k}=A \cap(A-k)$ with upper Banach density equal to $\delta_{k}$. Then the set $\left\{k \in \mathbb{Z}: \delta_{k}>\delta^{2}-\varepsilon\right\}$ is syndetic.

In order to prove the equivalence between reiteratively hypercyclic weighted shifts and frequently hypercyclic weighted shifts on $\ell^{p}$, we need a little bit more precise result. This result and its proof are a direct adaptation of Theorem 8 in [6] to sets with upper Banach density. We include here the proof of this adaptation for the sake of completeness.

Theorem 21. Let $A \subset \mathbb{Z}_{+}$with $\overline{B d}(A)=\delta>0, \varepsilon>0$ and $B_{k}=A \cap(A-k)$ where $k \geq 1$. Then there exist an increasing sequence $\left(m_{i}\right)_{i \geq 1} \subset \mathbb{N}$ and an increasing sequence $\left(k_{i}\right)_{i \geq 1} \subset \mathbb{N}$ such that

$$
\text { 1. } \frac{\mid A \cap\left[m_{i}, m_{i}+k_{i}[\mid\right.}{k_{i}} \underset{i \rightarrow \infty}{\longrightarrow} \delta \text {; }
$$


2. $\frac{\mid B_{k} \cap\left[m_{i}, m_{i}+k_{i}[\mid\right.}{k_{i}} \underset{i \rightarrow \infty}{\longrightarrow} \eta_{k}$ for some $\eta_{k}$;

3. the set $F:=\left\{k \in \mathbb{N}: \eta_{k}>(1-\varepsilon) \delta^{2}\right\}$ is syndetic.

Proof. Since $\overline{B d}(A)=\delta$, there exists an increasing sequence $\left(n_{i}\right)_{i \geq 1}$ such that

$$
\frac{\mid A \cap\left[n_{i}, n_{i}+i[\mid\right.}{i} \underset{i \rightarrow \infty}{\longrightarrow} \delta
$$

Since for any $k \geq 1$, the set $\left(\frac{\mid B_{k} \cap\left[n_{i}, n_{i}+i||\right.}{i}\right)_{i}$ belongs to the compact $[0,1]$, we can extract a subsequence $\left(n_{k_{i}}\right)_{i \geq 1}$ such that for any $k \geq 1,\left(\frac{\mid B_{k} \cap\left[n_{k_{i}}, n_{k_{i}}+k_{i}[\right.}{k_{i}}\right)_{i}$ converges to some $\eta_{k}$. We denote by $\left(m_{i}\right)_{i \geq 1}$ the sequence $\left(n_{k_{i}}\right)_{i \geq 1}$.

Let $R$ be a finite set such that for any $k, l \in R, k>l$, we have $\eta_{k-l} \leq(1-\varepsilon) \delta^{2}$. We consider $f(x):=|\{k \in R: x \in A-k\}|$. We deduce that for any $i \geq 1$

$$
\begin{aligned}
\sum_{x \in\left[m_{i}, m_{i}+k_{i}[\right.} f(x) & =\sum_{k \in R} \mid(A-k) \cap\left[m_{i}, m_{i}+k_{i}[\mid\right. \\
& =|R| \mid A \cap\left[m_{i}, m_{i}+k_{i}[\mid+O(1) .\right.
\end{aligned}
$$

and thus

$$
\frac{1}{k_{i}} \sum_{x \in\left[m_{i}, m_{i}+k_{i}[\right.} f(x) \underset{i \rightarrow \infty}{\longrightarrow}|R| \delta
$$

If we now consider the square of $f(x)$, we have

$$
f(x)^{2}=|\{k, l \in R: x \in(A-k) \cap(A-l)\}|=|\{k, l \in R: x+k \in A \cap(A+k-l)\}| .
$$

In the same way, we deduce that for any $i \geq 1$

$$
\begin{aligned}
& \sum_{x \in\left[m_{i}, m_{i}+k_{i}[\right.} f(x)^{2} \\
= & \sum_{k \in R} \mid A \cap\left[m_{i}, m_{i}+k_{i}\left[| + 2 \sum _ { k , l \in R , k > l } | B _ { k - l } \cap \left[m_{i}, m_{i}+k_{i}[\mid+O(1)\right.\right.\right. \\
= & |R| \mid A \cap\left[m_{i}, m_{i}+k_{i}\left[| + 2 \sum _ { k , l \in R , k > l } | B _ { k - l } \cap \left[m_{i}, m_{i}+k_{i}[\mid+O(1)\right.\right.\right.
\end{aligned}
$$

and thus

$$
\frac{1}{k_{i}} \sum_{x \in\left[m_{i}, m_{i}+k_{i}[\right.} f(x)^{2} \underset{i \rightarrow \infty}{\longrightarrow}|R| \delta+2 \sum_{k, l \in R, k>l} \eta_{k-l} \leq|R| \delta+(1-\varepsilon)|R|(|R|-1) \delta^{2} .
$$

We conclude that

$$
(|R| \delta)^{2} \leq|R| \delta+(1-\varepsilon)|R|(|R|-1) \delta^{2} .
$$

This inequality gives us the following condition on $|R|$ :

$$
|R| \leq \frac{1-\delta(1-\varepsilon)}{\delta \varepsilon}
$$

In other words, there exists a maximal finite set $R$ in the sense that for any $n \notin R$, there exists $k \in R$ such that $\eta_{n-k}>(1-\varepsilon) \delta^{2}$, i.e $n-k \in F$. We conclude that $F+R=\mathbb{Z}_{+}$ and in particular that $F$ is syndetic. 
Therefore, in the vein of [6, Corollary 9] and following essentially the same proof we obtain the following result:

Corollary 22. Let $A \subset \mathbb{Z}_{+}$be a set with positive upper Banach density and $\left(\alpha_{n}\right)_{n \in \mathbb{Z}}$ a sequence of non-negative real numbers such that $\sum_{n} \alpha_{n}=+\infty$. Suppose that there exist some $C>0$ and $N \in \mathbb{Z} \cup\{+\infty\}$ such that $\alpha_{n} \geq C \alpha_{n-1}$ for every $n<N$ and $\alpha_{n}=0$ for every $n \geq N$. If for any $n \in A$, we let

$$
\beta_{n}=\sum_{m \in A} \alpha_{m-n}
$$

then the sequence $\left(\beta_{n}\right)_{n \in A}$ is not bounded.

Proof. Let $\delta:=\overline{B d}(A)$ and $B_{k}=A \cap(A-k)$ for any $k \in \mathbb{Z}$. By Theorem 21, we know that there exist an increasing sequence $\left(m_{i}\right)_{i \geq 1} \subset \mathbb{N}$ and an increasing sequence $\left(k_{i}\right)_{i \geq 1} \subset \mathbb{N}$ such that for any $k \geq 1$

1. $\frac{\mid B_{k} \cap\left[m_{i}, m_{i}+k_{i}[\mid\right.}{k_{i}} \underset{i \rightarrow \infty}{\longrightarrow} \eta_{k}$ for some $\eta_{k}$;

2. the set $F:=\left\{k \geq 1: \eta_{k}>\frac{1}{2} \delta^{2}\right\}$ is syndetic.

We remark that for any $k \geq 1$, we have $B_{-k}=B_{k}+k$ and thus

$$
\frac{\mid B_{-k} \cap\left[m_{i}, m_{i}+k_{i}[\mid\right.}{k_{i}}=\frac{\mid B_{k} \cap\left[m_{i}-k, m_{i}+k_{i}-k[\mid\right.}{k_{i}} \underset{i \rightarrow \infty}{\longrightarrow} \eta_{k} .
$$

On the other hand, if we let $F_{\mathbb{Z}}=(-F) \cup F$, we deduce that the set $F_{\mathbb{Z}}$ is syndetic. Let $\left(f_{j}\right)_{j \in \mathbb{Z}}$ be an increasing enumeration of $F_{\mathbb{Z}}$ and $M \geq 1$ such that $f_{j}-f_{j-1} \leq M$ for any $j \in \mathbb{Z}$. We deduce that for any $f_{j}<N$, we have

$$
\alpha_{f_{j}} \geq \frac{\min \left(1, C^{M}\right)}{M} \sum_{f_{j-1}<i \leq f_{j}} \alpha_{i}
$$

and thus

$$
\sum_{n \in F_{\mathbb{Z}}} \alpha_{n}=\sum_{j: f_{j}<N} \alpha_{f_{j}} \geq \frac{\min \left(1, C^{M}\right)}{M} \sum_{n<N-M} \alpha_{n}=\frac{\min \left(1, C^{M}\right)}{M}\left(\sum_{n \in \mathbb{Z}} \alpha_{n}-\sum_{n=N-M}^{N-1} \alpha_{n}\right)=\infty,
$$

where we consider $\sum_{n=N-M}^{N-1} \alpha_{n}=0$ if $N=+\infty$.

We then consider the sequence $\left(s_{i}\right)_{i \geq 1}$ defined by

$$
s_{i}=\sum_{n \in A \cap\left[m_{i}, m_{i}+k_{i}[\right.} \beta_{n}=\sum_{\substack{n \in A \cap\left[m_{i}, m_{i}+k_{i}[ \\m \in A\right.}} \alpha_{m-n}
$$

If we arrange this sum according to the value $k=m-n$ and if we keep only the terms where $k \in F_{\mathbb{Z}}$, then we get for any $l \geq 1$,

$$
s_{i} \geq \sum_{k \in F_{\mathbb{Z}},|k|<l} \alpha_{k} \mid B_{k} \cap\left[m_{i}, m_{i}+k_{i}[\mid .\right.
$$


We deduce that for any $l \geq 1$

$$
\begin{aligned}
\limsup _{i \rightarrow \infty} \frac{1}{k_{i}} \sum_{n \in A \cap\left[m_{i}, m_{i}+k_{i}[\right.} \beta_{n} & =\limsup _{i \rightarrow \infty} \frac{s_{i}}{k_{i}} \\
& \geq \sum_{k \in F_{\mathbb{Z}},|k|<l} \alpha_{k} \lim _{i \rightarrow \infty} \frac{\mid B_{k} \cap\left[m_{i}, m_{i}+k_{i}[\mid\right.}{k_{i}} \\
& \geq \sum_{k \in F_{\mathbb{Z}},|k|<l} \alpha_{k} \eta_{|k|} \geq \frac{\delta^{2}}{2} \sum_{k \in F_{\mathbb{Z}},|k|<l} \alpha_{k} .
\end{aligned}
$$

Since $\sum_{n \in F_{\mathbb{Z}}} \alpha_{n}=+\infty$, we conclude that the sequence $\left(\beta_{n}\right)_{n \in A}$ cannot be bounded.

We can now prove the desired equivalence for weighted shifts on $\ell^{p}$.

Theorem 23. Let $B_{w}$ be a weighted shift on $\ell^{p}(\mathbb{Z})$ or on $\ell^{p}\left(\mathbb{Z}_{+}\right)$with $1 \leq p<\infty$. Then $B_{w}$ is reiteratively hypercyclic if and only if $B_{w}$ is frequently hypercyclic.

Proof. We only prove this equivalence in the case of weighted shifts on $\ell^{p}(\mathbb{Z})$ since the case of weighted shifts on $\ell^{p}\left(\mathbb{Z}_{+}\right)$is similar and easier.

Let $B_{w}$ be a reiteratively hypercyclic weighted shift on $\ell^{p}(\mathbb{Z})$. There exists a vector $x \in \ell^{p}\left(\mathbb{Z}_{+}\right)$such that the set

$$
A:=\left\{n \in \mathbb{Z}_{+}:\left\|B_{w}^{n} x-e_{0}\right\| \leq \frac{1}{2}\right\}
$$

has a positive upper Banach density. For any $n \in A$, we remark that we have $\mid w_{1} \cdots w_{n} x_{n}-$ $1 \mid \leq \frac{1}{2}$ and

$$
\begin{aligned}
\frac{1}{2^{p}} & \geq \sum_{m<n}\left|w_{m-n+1} \cdots w_{0} w_{1} \cdots w_{m}\right|^{p}\left|x_{m}\right|^{p}+\sum_{m>n}\left|w_{m-n+1} \cdots w_{m}\right|^{p}\left|x_{m}\right|^{p} \\
& =\sum_{m<n}\left|w_{m-n+1} \cdots w_{0}\right|^{p}\left|w_{1} \cdots w_{m} x_{m}\right|^{p}+\sum_{m>n} \frac{\left|w_{1} \cdots w_{m} x_{m}\right|^{p}}{\left|w_{1} \cdots w_{m-n}\right|^{p}} \\
& \geq \sum_{m<n, m \in A}\left|w_{m-n+1} \cdots w_{0}\right|^{p}\left|w_{1} \cdots w_{m} x_{m}\right|^{p}+\sum_{m>n, m \in A} \frac{\left|w_{1} \cdots w_{m} x_{m}\right|^{p}}{\left|w_{1} \cdots w_{m-n}\right|^{p}} \\
& \geq \frac{1}{2^{p}}\left(\sum_{m<n, m \in A}\left|w_{m-n+1} \cdots w_{0}\right|^{p}+\sum_{m>n, m \in A} \frac{1}{\left|w_{1} \cdots w_{m-n}\right|^{p}}\right) .
\end{aligned}
$$

We get for any $n \in A$

$$
\sum_{m<n, m \in A}\left|w_{m-n+1} \cdots w_{0}\right|^{p} \leq 1 \text { and } \sum_{m>n, m \in A} \frac{1}{\left|w_{1} \cdots w_{m-n}\right|^{p}} \leq 1 .
$$

Thanks to Corollary 22, we can deduce from (18) the convergence of series $\sum_{n \geq 1} \frac{1}{\left|w_{0} \cdots w_{n}\right|^{p}}$ and $\sum_{n<0}\left|w_{n} \cdots w_{0}\right|^{p}$. Indeed, if we let $\alpha_{n}=0$ for any $n \leq 0$ and $\alpha_{n}=\frac{1}{\left|w_{1} \cdots w_{n}\right|^{p}}$ for any $n \geq 1$, we have $\alpha_{n} \geq C \alpha_{n-1}$ where $C=\inf \left\{\left|w_{n}\right|^{-p}: n \geq 1\right\}$ is strictly positive since $w$ is bounded. Therefore, if $\sum_{n \geq 1} \frac{1}{\left|w_{1} \cdots w_{n}\right|^{p}}=\infty$, we deduce from Corollary 22 with $N=+\infty$ that the sequence $\left(\beta_{n}\right)_{n \in A}$ is unbounded where

$$
\beta_{n}:=\sum_{m \in A} \alpha_{m-n}=\sum_{m>n, m \in A} \alpha_{m-n}=\sum_{m>n, m \in A} \frac{1}{\left|w_{1} \cdots w_{m-n}\right|^{p}} .
$$


This is a contradiction with (18).

On the other hand, if we let $\alpha_{n}=0$ for any $n \geq 0$ and $\alpha_{n}=\left|w_{n+1} \cdots w_{0}\right|^{p}$ for any $n<0$, we have $\alpha_{n} \geq C \alpha_{n-1}$ for any $n<0$ where $C=\inf \left\{\left|w_{n}\right|^{-p}: n \leq-1\right\}$. As previously, we deduce from Corollary 22 with $N=0$ that if $\sum_{n<0}\left|w_{n} \cdots w_{0}\right|^{p}=\infty$ then the sequence $\left(\sum_{m<n, m \in A}\left|w_{m-n+1} \cdots w_{0}\right|^{p}\right)_{n \in A}$ is unbounded which is a contradiction with (18).

Finally, we get the desired result since the convergence of series $\sum_{n \geq 1} \frac{1}{\left|w_{0} \cdots w_{n}\right|^{p}}$ and $\sum_{n<0}\left|w_{n} \cdots w_{0}\right|^{p}$ implies that $B_{w}$ is frequently hypercyclic ([6, Theorem 3])

This equivalence for weighted shifts on $\ell^{p}\left(\mathbb{Z}_{+}\right)$implies that there exists some mixing operator which is not reiteratively hypercyclic. Indeed, we know that a weighted shift $B_{w}$ on $\ell^{p}\left(\mathbb{Z}_{+}\right)$is mixing if and only if $\prod_{k=1}^{\infty}\left|w_{k}\right|$ tends to infinity [11]. Therefore, the weighted backward shift $B_{w}$ with $w_{n}=((n+1) / n)^{\frac{1}{p}}$ is a mixing operator on $\ell^{p}\left(\mathbb{Z}_{+}\right)$which is not frequently hypercyclic and thus not reiteratively hypercyclic (Theorem 23 ).

Theorem 24. There exists some mixing operator which is not reiteratively hypercyclic.

Thanks to Grosse-Erdmann and Peris [14], we know that every frequently hypercyclic operator is weakly mixing. We observe that every reiteratively hypercyclic operator is topologically ergodic (thus, weakly mixing [15]). See also [1].

Proposition 25. Let $X$ be a separable $F$-space and $T \in \mathcal{L}(X)$. If $T$ is reiteratively hypercyclic, then $T$ is topologically ergodic.

Proof. Let $U, V$ non-empty open sets in $X$ and $n \in N(U, V)$. We consider the non-empty open set $U_{n}:=U \cap T^{-n}(V)$. Let $x \in X$ such that $\overline{B d}\left(N\left(x, U_{n}\right)\right)>0$. We remark that

$$
N\left(x, U_{n}\right)-N\left(x, U_{n}\right)+n \subseteq N(U, V) .
$$

Indeed, if $s_{1}, s_{2} \in N\left(x, U_{n}\right)$, then

$$
T^{s_{2}} x \in U \quad \text { and } \quad T^{s_{1}-s_{2}+n}\left(T^{s_{2}} x\right)=T^{n}\left(T^{s_{1}} x\right) \in V
$$

On the other hand, we know that if $A$ is a set with positive upper Banach density, then $A-A$ is syndetic [12, Proposition 3.19]. We conclude that $N\left(x, U_{n}\right)-N\left(x, U_{n}\right)$ is syndetic and thus $N(U, V)$ is also syndetic.

\section{References}

[1] C. Badea and S. Grivaux, Unimodular eigenvalues, uniformly distributed sequences and linear dynamics, Advances in Math. 211 (2007) 766-793.

[2] F. Bayart and S. Grivaux, Frequently hypercyclic operators, Trans. Amer. Math. Soc. 358 (2006), 5083-5117.

[3] F. Bayart and S. Grivaux, Invariant Gaussian measures for operators on Banach spaces and linear dynamics, Proc. Lond. Math. Soc. (3) 94 (2007), 181-210.

[4] F. Bayart and É. Matheron, Dynamics of linear operators, Cambridge Tracts in Mathematics, No. 179, 2009.

[5] F. Bayart and É. Matheron, (Non-)weakly mixing operators and hypercyclicity sets, Ann. Inst. Fourier (Grenoble) 59 (2009), 1-35. 
[6] F. Bayart and I. Ruzsa, Difference sets and frequently hypercyclic weighted shifts, Ergod. Th. \& Dynam. Sys. (2013), available on CJO2013. doi:10.1017/etds.2013.77.

[7] V. Bergelson, T. Downarowicz, Large sets of integers and hierarchy of mixing properties of measure-preserving systems, Colloq. Math. 110 (2008), no. 1, 117-150.

[8] L. Bernal-González and K.-G. Grosse-Erdmann, The Hypercyclicity Criterion for sequences of operators, Studia Math. 157 (2003), 17-32.

[9] J. Bès and A. Peris, Hereditarily hypercyclic operators, J. Funct. Anal. 167 (1999), 94-112.

[10] A. Bonilla and K.-G. Grosse-Erdmann, Frequently hypercyclic operators and vectors, Ergodic Theory Dynam. Systems 27 (2007), 383-404. Erratum: Ergodic Theory Dynam. Systems 29 (2009), 1993-1994.

[11] G. Costakis and M. Sambarino, Topologically mixing hypercyclic operators, Proc. Amer. Math. Soc. 132 (2004), 385-389.

[12] H. Furstenberg, Recurrence in ergodic Theory and combinatorial number Theory, Princeton university press, Princeton, N.J., 1981.

[13] K.-G. Grosse-Erdmann Hypercyclic and chaotic weighted shifts, Studia Math. 139 (2000), 47-68

[14] K.-G. Grosse-Erdmann and A. Peris, Frequently dense orbits, C. R. Math. Acad. Sci. Paris 341 (2005), 123-128.

[15] K.-G. Grosse-Erdmann and A. Peris, Weakly mixing operators on topological vector spaces, RACSAM 104 (2), 2010, 413-426.

[16] K.-G. Grosse-Erdmann and A. Peris Manguillot, Linear chaos, Universitext, Springer, London, 2011.

[17] M. Pollicott, K. Schmidt, Ergodic theory of $\mathbb{Z}^{d}$-actions, London Math. Soc. Lecture Note Series 228 .

[18] H. N. Salas, Hypercyclic weighted shifts, Trans. Amer. Math. Soc. 347 (1995), 9931004.

[19] T. Salat and V. Toma, A classical Olivier's theorem and statistical convergence, Ann. Math. Blaise Pascal 10 (2003), 305-313.

[20] S. Shkarin, On the spectrum of frequently hypercyclic operators, Proc. Amer. Math. Soc. 137 (2009), 123-134. 